\title{
Contrast-enhanced MR angiography
}

\author{
J. H. Maki, T. L. Chenevert, M. R. Prince \\ Department of Radiology, Division of MRI, University of Michigan, 1500 E. Medical Center Drive, Ann Arbor, MI 48109-0030, USA
}

Recent advances in the implementation and understanding of three-dimensional (3D) contrast magnetic resonance angiography (MRA) are making vascular imaging throughout the body safer and more accurate. This article is intended to describe the basic principles behind 3D contrast MRA and demonstrate how to apply this technique to multiple vascular territories with emphasis on abdominal applications. The inherent advantages of 3D contrast MRA over "conventional" MRA techniques such as time of flight (TOF) and phase contrast (PC) are discussed. In addition, techniques for improved spatial and temporal resolution, decreased respiratory motion artifacts, decreased contrast dosing, and more accurate bolus timing are explored.

\section{Conventional MRA}

Magnetic resonance pulse sequences can exploit blood motion to visualize vascular structures directly without the use of intravascular contrast material. In 2D or 3D TOF angiography, which is usually performed using a flow compensated gradient refocused sequence, stationary tissues are "saturated" and thus have low signal intensity [1-3]. Blood upstream of the imaging volume, however, is ' "unsaturated.' When this blood flows into the imaging volume, it is bright compared with the stationary background tissues. This "inflow" or TOF approach often works well, especially in normal arteries and veins, including the extremities, carotid arteries, and cerebral vasculature [4-13]. It also has the benefit of allowing selective saturation of blood adjacent to the imaging volume such that either arterial or venous signal can be suppressed. One major disadvantage of TOF angiography is inplane saturation, which can be a problem with slowly flowing and tortuous arteries or when the long axis of the vessel coincides with the scan plane. A second drawback is turbulence-induced signal loss in

Correspondence to: J. H. Maki, Department of Radiology (114), Puget Sound Veterans Administration Hospital, 1660 South Columbian Way, Seattle, WA 98108, USA a region of stenosis $[14,15]$. In addition, imaging times tend to be long, in part because it is often necessary to image perpendicular to the vessel axis to avoid inplane saturation.

PC angiography uses phase shifts as blood flows in the presence of "flow-encoding"' gradients [1, 16, 17]. Using phase difference images, signal phase intensity is proportional to velocity, and stationary background tissue is suppressed. The flow encoding gradients can be applied in any or multiple directions depending on the desired flow sensitivity. PC angiography can be implemented as a 2D or 3D gradient refocused sequence. It has proved useful for evaluation of the renal arteries, carotids, and portal vein [8, 12, 17-21]. In normal vessels without stenoses or turbulence, the results are often spectacular.

Another strength of PC angiography is its ability to measure flow velocities. In combination with CINE and cardiac gating, a time-resolved velocity profile can be generated that is quite similar to an ultrasound Doppler waveform $[22,23]$. This profile provides physiologic flow data and allows the quantitative measurement of flow rates. Unfortunately, it is this same velocity sensitivity that limits PC angiography. Strong flow encoding gradients make the sequence susceptible to degradation from bulk (cardiac, respiratory, translational) motion. In addition, PC sequences are only sensitive to a specified range of velocities [20]. In occlusive disease, turbulence causes a broad spectrum of rapidly changing velocities, which in turn causes intravoxel phase dispersion and signal loss. Thus, artifactual loss of vessel visualization at a stenosis is common [14]. Interestingly, this apparent deficiency can be useful for identifying regions of turbulent, disordered flow when used in conjunction with 3D contrast MRA.

\section{Contrast-enhanced MRA: theory}

Three-dimensional contrast-enhanced MRA is performed in a manner analogous to conventional contrast angiography. Instead of relying on blood motion to create intravascular signal, a contrast agent (gadolinium chelate) is introduced to change the T1 (spin lattice) relaxation of blood such that it is very different from surrounding tissues. Blood 
can then be directly imaged irrespective of flow, which alleviates many of the problems inherent to TOF and PC angiography. In particular, sensitivity to turbulence is dramatically reduced, and inplane saturation effects are eliminated $[14,15]$. The technique allows a small number of slices oriented in the plane of the vessels of interest to image an extensive region of vascular anatomy (equal to the field of view) in a short period of time. Thus, $3 \mathrm{D}$ contrast MRA is intrinsically fast, with high-quality breath-hold angiograms being possible when using modern MR units.

\section{Gadolinium chelates}

Gadolinium $\left(\mathrm{Gd}^{3+}\right)$ is a paramagnetic metal ion that decreases both the spin-lattice (T1) and spin-spin (T2) relaxation times [24]. Because $\mathrm{Gd}^{3+}$ itself is biologically toxic, it is chelated with ligands such as DTPA (gadopentetate dimeglumine) to form small-molecularweight contrast agents [25]. These "extracellular" agents diffuse from the intravascular compartment into the interstitial space in a matter of minutes, meaning selective imaging of the vascular structures must be performed rapidly $[14,25]$. As compared with iodinated contrast agents, gadolinium chelates have a very low rate of adverse events and no nephrotoxicity, a significant advantage when evaluating patients with impaired renal function [26-30]. Gadolinium chelates decrease the $\mathrm{T} 1$ of blood according to the equation $[15,31]$.

$$
1 / \mathrm{T} 1=1 / 1200+\mathrm{R}_{1}[\mathrm{Gd}]
$$

where $\mathrm{R}_{1}$ is the field-dependent $\mathrm{T} 1$ relaxivity of the gadolinium chelate (approximately $4.5 \mathrm{~s}^{-1} \cdot \mathrm{mmol}^{-1}$ at $1.5 \mathrm{~T}$ ), [Gd] is the concentration of gadolinium chelate, and $1200(\mathrm{~ms})$ is the T1 of blood without gadolinium (at $1.5 \mathrm{~T}$ ). A similar equation exists for $\mathrm{T} 2$ (which is always shorter than T1).

\section{“'Blood pool' agents}

Because of the rapid redistribution of gadolinium chelates into the extracellular compartment, some investigators have suggested using intravascular paramagnetic contrast agents rather than "extracellular" gadolinium chelates for 3D contrast MRA. These agents include highmolecular-weight polychelates such as albumin-(Gd-DTPA) $)_{x}$, polylysine-(Gd-DTPA) $)_{\mathrm{x}}$, dextran-(Gd-DTPA) $)_{\mathrm{x}}$, and superparamagnetic iron oxides [32-38]. The potential advantages of these agents include a long intravascular half-life with minimal leakage into the interstitial space and significantly higher relaxivities $\left(\mathrm{R}_{1}\right)$, allowing decreased molar dosing.

To date, with the exception of a couple of human MRA studies using superparamagnetic iron oxides, most of the investigation has been in animals, with much work aimed at evaluating relative tissue blood volume, estimating perfusion, and evaluating capillary integrity [37, 39-43]. It is not yet known whether these blood pool agents will have any advantage over extracellular agents for dynamic arterialphase MRA. There are also concerns regarding the safety profiles of some of these agents [44-46]. Although MR angiography using blood pool agents may ultimately be useful, current clinical work uses extracellular gadolinium chelates.

\section{Pulse sequence}

Gadolinium-enhanced MRA (Gd-MRA) exploits the transient shortening in blood $\mathrm{T} 1$ following intravenous administration of gadolinium chelates using a fast 3D spoiled gradient echo (SPGR) sequence [15, $47,48]$. We use the term "transient" because this technique exploits the "first pass" of gadolinium and because studies have indicated that $80 \%$ of the administered gadolinium dose disappears from the intravascular space within 5 min [49]. With this technique, typical repetition times (TR) are less than $10 \mathrm{~ms}$, with echo times (TE) of 1-3 ms and total scan times in the 20-45-s range. This type of sequence is ideally suited to MRA because it has high spatial resolution and an intrinsically high SNR, it is fast (it can be performed in a breath-hold), and it can be oriented and reformatted in any desired plane $[13,50$ 54]. In addition, because intravascular signal is dependent on $\mathrm{T} 1 \mathrm{re}-$ laxation rather than on inflow or phase accumulation, inplane saturation and turbulence induced signal loss are not a problem [15]. The relative signal intensity (SI) for a 3D SPGR acquisition can be written as [55]

$$
\mathrm{SI}=\left[\mathrm{N}(\mathrm{H})\left(1-\mathrm{e}^{-\mathrm{TR} / \mathrm{T} 1}\right) /\left(1-\cos (\alpha) \mathrm{e}^{-\mathrm{TR} / \mathrm{T} 1}\right)\right] \sin (\alpha) \mathrm{e}^{-\mathrm{TE} / \mathrm{T} 2^{*}}
$$

where $\mathrm{N}(\mathrm{H}), \mathrm{TR}, \mathrm{TE}$, and $\alpha$ are the proton density, repetition time, echo time, and flip angle, respectively. Examining equation 2, SI is maximized if either TR is very long or T1 is very short because as (TR/T1) becomes large, $\mathrm{e}^{-\mathrm{TR} / \mathrm{T} 1}$ approaches zero. Of these options, lengthening TR is not desirable because it increases scan time. This leaves shortening $\mathrm{T} 1$ as the primary method for increasing intravascular signal intensity. Figure 1 shows an example of relative signal intensity versus flip angle $\alpha$ for different T1 values (assuming a TR of $7 \mathrm{~ms}$ with $\mathrm{TE} \ll \mathrm{T} 2 *)$. Note the significant increase in signal intensity as T1 approaches zero.

\section{Gadolinium dose and T1}

For blood to appear bright with respect to the background tissues, sufficient gadolinium must be administered to reduce the blood T1 below the $\mathrm{T} 1$ of the brightest background tissue, i.e., fat, which has a $\mathrm{T} 1$ of approximately $270 \mathrm{~ms}$. Ignoring gadolinium recirculation and assuming that the blood compartment constitutes $8 \%$ of body mass and the extracellular fluid compartment is $30 \%$ of body mass, the equilibrium $\mathrm{T} 1$ of blood can be calculated from equation 1 for a given dose of gadolinium, both before and after redistribution into the extracellular compartment [14] (Fig. 2).

Based on Figure 2, doses of greater than approximately 0.1 and $0.2 \mathrm{mmol} / \mathrm{kg}$ are required to decrease the blood T1 to below that of fat for blood pool and redistribution phases respectively, but Gd-MRA is typically performed dynamically rather than at equilibrium. This method has the advantage of eliminating steady-state background signal but at the same time is essentially a "one-shot" technique, meaning that once gadolinium is given and dynamic imaging performed, the process cannot be repeated until gadolinium is cleared from the soft tissues, a period of many hours considering the biologic half-life of approximately $90 \mathrm{~min}$ [56]. Hence, timing is extremely important in dynamic Gd-MRA. To appreciate timing issues, the relationship between injection rate, blood T1, and resultant signal intensity must be understood.

For times shorter than the recirculation time, gadolinium concentration of the bolus in blood can be approximated from equation 3 [14, 15]:

$$
[\mathrm{Gd}]=\frac{(\text { rate of Gd injection in } \mathrm{mol} / \mathrm{L})}{(\text { cardiac output in } \mathrm{L} / \mathrm{s})}
$$

Combining equations 1 and 3 and assuming no recirculation, blood $\mathrm{T} 1$ can be calculated for a given cardiac output and injection rate, which is demonstrated in Figure 3 for cardiac outputs of 3, 5, and 7 $\mathrm{L} / \mathrm{min}$ using a standard gadolinium chelate preparation with a concentration of $0.5 \mathrm{~mol} / \mathrm{L}$.

Note from Figure 3 that for a "typical" cardiac output of $5 \mathrm{~L} / \mathrm{min}$, an injection rate of $1 \mathrm{cc} / \mathrm{s}$ yields a blood T1 of $36 \mathrm{~ms}$, whereas a $2 \mathrm{cc} /$ $\mathrm{s}$ injection produces a blood $\mathrm{T} 1 \mathrm{of} 18 \mathrm{~ms}$. These $\mathrm{T} 1$ values are much shorter than the T1 values of fat. Of course, such a fast injection cannot and need not be sustained for the full imaging time. For a typical $70-\mathrm{kg}$ patient receiving a "double dose" $(0.2 \mathrm{mmol} / \mathrm{kg})$ of gadolinium, this amounts to $28 \mathrm{cc}$ of a $0.5 \mathrm{~mol} / \mathrm{L}$ gadolinium chelate preparation. Thus, a $1 \mathrm{cc} / \mathrm{s}$ injection can be sustained for $28 \mathrm{~s}$, or a $2 \mathrm{cc} / \mathrm{s}$ injection sustained for $14 \mathrm{~s}$. Even though the imaging time may be longer than the injection time, by properly timing the bolus with respect to image acquisition, full advantage of the dynamic blood T1 shortening can be obtained because of the unique way in which MR data are acquired. 

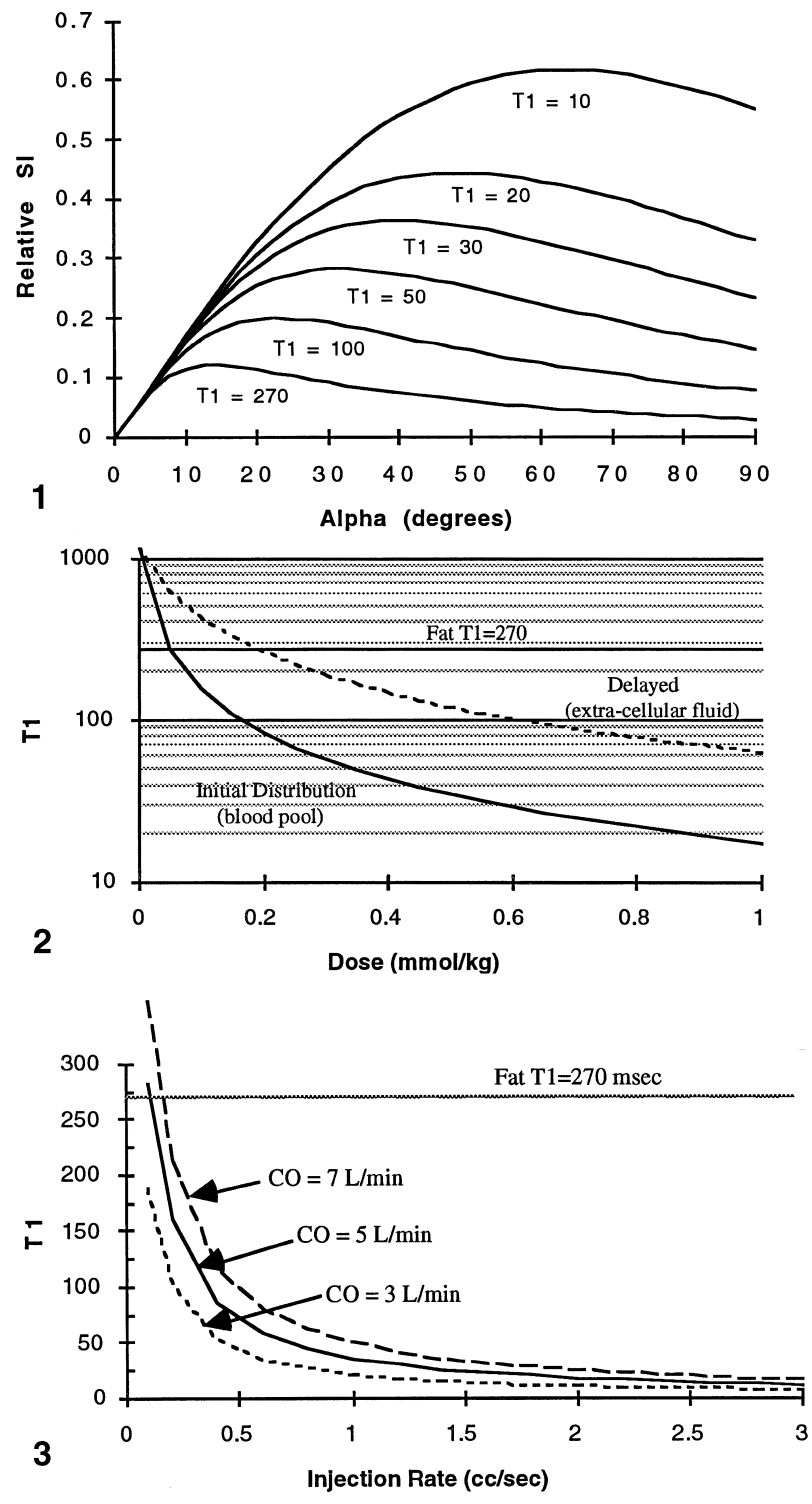

Fourier (k-space) considerations

MR imaging does not map spatial data linearly with respect to time. With 3D MR imaging, the entire 3D "Fourier" or "k-space" data set is collected before individual slices are reconstructed. Because k-space maps spatial frequencies rather than spatial data itself, the $\mathrm{k}$-space data do not directly correspond to image space. Instead, different parts of k-space data determine different image features. For example, the center of k-space, or "low" spatial frequencies, dominate image contrast, whereas the periphery of k-space, or "high" spatial frequencies, contribute more to fine details such as edges [57, 58], which means that the state of the intravascular T1 in large vessels is essentially "captured" at the time corresponding to acquisition of central k-space [57]. If central k-space is collected when arterial [Gd] is high but gadolinium has not yet reached the venous structures (arterial phase), only the arteries will be bright. The fact that intravascular gadolinium concentration may not be uniform over the entire acquisition time may generate artifacts, but these are relatively minor provided that [Gd] is not rapidly changing over the critical central portion of k-space [57]. Thus, with proper timing, gadolinium injection duration need not be
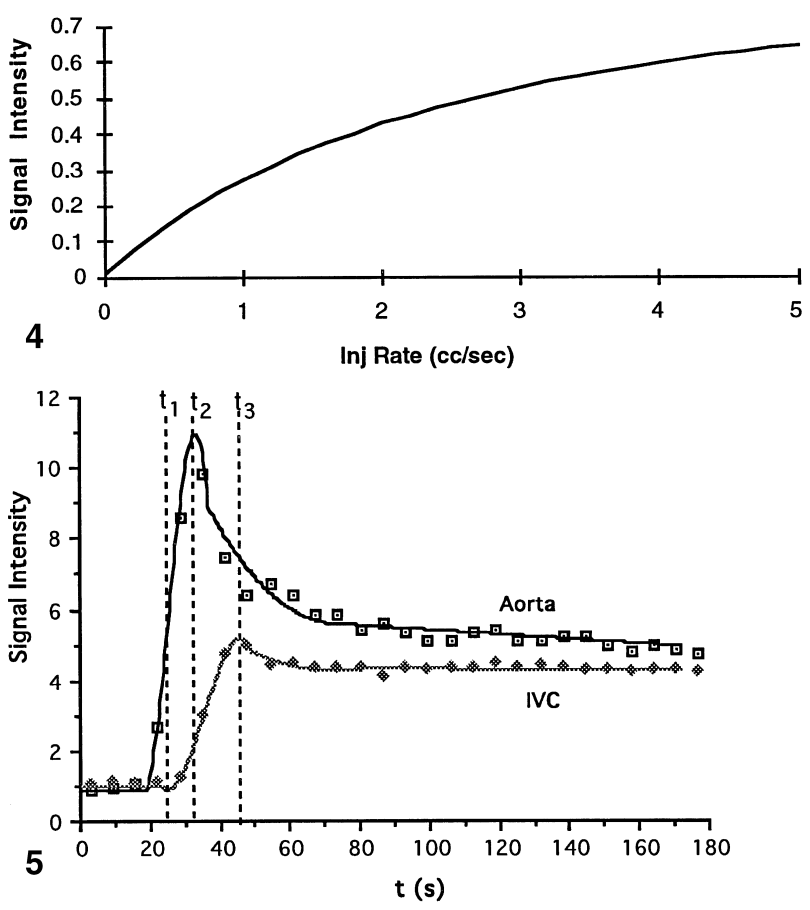

Fig. 1. Relative signal intensity for different $T 1$ values versus flip angle $\alpha$ for an SPGR sequence. TR $=7 \mathrm{~ms}$, TE $\ll \mathrm{T} 2 *$.

Fig. 2. Blood T1 versus gadolinium dose. Initial distribution (blood pool) and delayed phases.

Fig. 3. Blood $\mathrm{T} 1$ versus injection rate for changing cardiac outputs (CO), assuming no recirculation.

Fig. 4. Relative signal intensity versus gadolinium injection rate for an SPGR sequence. Flip angle $\alpha=55^{\circ}$, cardiac output $=5 \mathrm{~L} / \mathrm{min}$.

Fig. 5. Relative signal intensity in the aorta and inferior vena cava (IVC) versus time following a $0.1-\mathrm{mmol} / \mathrm{kg}$ gadopentatate dimeglumine injection of $10 \mathrm{~s}$ duration. Keyhole SPGR technique. Adapted from the work of Strouse et al. [67].

as long as the entire acquisition time. For a given gadolinium dose, the injection strategy then becomes a trade off between a fast injection (shorter T1, more intravascular signal) and long injection (more uniform $\mathrm{T} 1$, fewer artifacts).

Another factor to be considered is phase-encoding order. Traditionally, phase encoding is performed "sequentially" such that central $\mathrm{k}$-space is acquired at the mid-point of the scan. Alternatively, phase encoding can be performed in a "centric" fashion such that central $\mathrm{k}$-space is acquired at the beginning of the scan $[59,60]$. Although centric phase encoding order can be somewhat more prone to artifacts if [Gd] is rapidly changing during central $\mathrm{k}$-space acquisition, it greatly simplifies bolus timing and is less susceptible to incomplete breath-holds [57, 61, 62].

\section{Contrast material injection rate}

For injection rates greater than approximately $2 \mathrm{cc} / \mathrm{s}$ (Fig. 3), there is diminishing return with respect to T1 shortening. But even small decreases in T1 lead to tremendous increases in signal intensity (Fig. 1). 

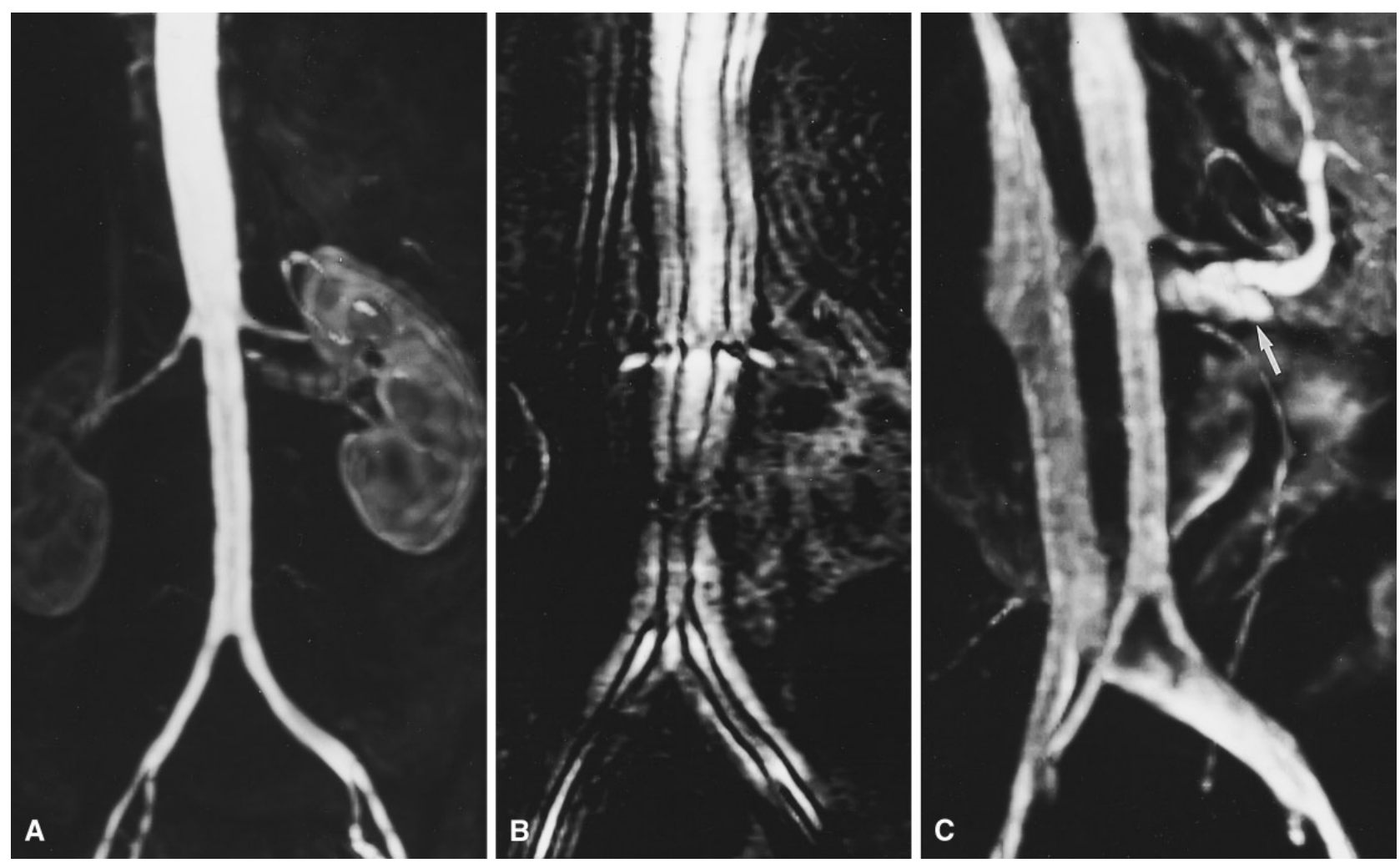

Fig. 6. MIP images from coronal 3D gadolinium-enhanced MRAs of the abdominal aorta in the same patient. A Optimal bolus timing for arterial phase. B Acquisition of central k-space too early, with resul- tant "ringing" artifact. C Delayed arteriovenous phase. Note the surgically created portal vein to the left renal vein shunt (arrow).
Combining equations $1-3$ and assuming $\mathrm{TE} \ll \mathrm{T} 2 *$, signal intensity versus injection rate can be plotted for a fixed cardiac output and flip angle $\left(5 \mathrm{~L} / \mathrm{min}\right.$ and $55^{\circ}$ in this example; (Fig. 4). This graph demonstrates that signal intensity increases asymptotically as injection rate increases, with only minimal increases seen beyond a rate of $4-5 \mathrm{cc} / \mathrm{s}$.

Different investigators have taken different approaches to optimizing injection rate. Before the advent of high-speed gradient systems, which permitted total acquisition times of less than $1 \mathrm{~min}$ (in the realm of a breath-hold), imaging times were on the order of 3-5 min, and injections were typically performed so that a "double dose" $(0.2 \mathrm{mmol} / \mathrm{kg})$ was administered at a uniform rate over the entire scan duration [47, 63]. For a 4-min scan on a 70-kg patient, this amounted to an injection rate of just over $0.1 \mathrm{cc} / \mathrm{s}$. Many investigators now use a "double dose" $(0.2 \mathrm{mmol} /$ $\mathrm{kg}$ ) of gadolinium chelate with injection rates of $1-2 \mathrm{cc} / \mathrm{s}$, whereas some use up to a "quadruple dose" $(0.4 \mathrm{mmol} / \mathrm{kg})$ and rates of $4-5 \mathrm{cc} / \mathrm{s}[13$, $52,54,64,65]$. We personally feel that "double dose" with an injection rate of about $2 \mathrm{cc} / \mathrm{s}$ is a good compromise between maximal arterial signal and minimal artifacts for breath-hold scans [66]

\section{Contrast-enhanced MRA: bolus timing considerations}

The timing of the gadolinium bolus acquisition of central k-space is extremely important. The contrast travel time, defined as the time required for contrast to travel from the injection site to the vascular territory of interest, is highly variable. Some idea of contrast travel times and arterial/ venous enhancement profiles can be gained by examining the work of Strouse et al., who evaluated signal change in the aorta and IVC based on rapid time resolved 3D SPGR "keyhole", imaging [67]. An example of this is shown in Figure 5, where data are averaged from four patients receiving a $0.1 \mathrm{mmol} / \mathrm{kg}$ bolus of gadopentatate dimeglumine over $10 \mathrm{~s}$ (approximately $1.5 \mathrm{cc} / \mathrm{s}$ bolus, beginning at time $\mathrm{t}=0$ ).

First note that arterial signal (proportional to [Gd]) peaks rather sharply, with an approximately $8-10$-s delay before subsequent venous enhancement. (If the bolus were of longer duration, i.e., larger total dose, the arterial peak would be somewhat broader.) This observation has important implications. Because intravascular signal intensity is determined by the gadolinium concentration at the time the center of $\mathrm{k}$-space is collected [57], whether central $\mathrm{k}$-space is acquired at time $t_{1}, t_{2}$, or $t_{3}$ (see Figure 5) has a dramatic influence over the resultant image. Perfect timing $\left(\mathrm{t}_{2}\right)$ yields maximum arterial signal with minimal venous signal (Fig. 6A). If, however, central k-space data are acquired too early $\left(\mathrm{t}_{1}\right.$; while arterial [Gd] is still rapidly increasing), severe "ringing" or "banding" artifacts can be generated (Fig. 6B) [57]. These artifacts also are referred to as "leading edge", artifacts because they result from imaging during the leading edge of bolus arrival. Acquiring central k-space data too late $\left(\mathrm{t}_{3}\right)$ leads to submaximal arterial signal intensity and associated venous enhancement. These two factors can be detrimental to evaluation of the arterial structures. An example of such delayed timing is shown in Figure 6C. Hence, if one knows or can predict or measure the time course of intravascular [Gd], the placement of central k-space can be tailored to achieve the desired image characteristics, be it maximum arterial signal, maximum portal venous signal, etc.

\section{“Best-guess"' technique}

Several solutions to proper bolus timing have been offered. Perhaps the simplest is to just make an educated "best guess.'” The best guess 
involves estimating the contrast travel time from the site of injection to the vascular structure of interest. Although this time is highly variable (in a study of 50 patients, mean time between the initiation of gadolinium injection and arrival in the abdominal aorta was $23 \pm 5$ $\mathrm{s}$, with a range of 13-37 s), an experienced MR angiographer can reliably achieve good results by taking factors such as injection site, patients age, cardiac output, and vascular anatomy into consideration [69]. Using this technique with a sequential phase encoding order, timing can be calculated as follows [52]:

imaging delay $=($ estimated contrast travel time $)$

$$
+ \text { (injection time/2) - (imaging time/2) }
$$

where imaging delay is the delay between initiating the bolus and starting the scan (if negative, it refers to the delay between initiating the scan and starting the bolus). Equation 4 times things such that the midpoint of the bolus arrives at the desired vascular territory at the midpoint (central k-space) of the scan. As an example, for a scan time of $30 \mathrm{~s}$ with an estimated contrast travel time of $20 \mathrm{~s}$ and a $28-\mathrm{cc}$ gadolinium bolus at $2 \mathrm{cc} / \mathrm{s}$ (double dose for a 70-kg patient with a 14$\mathrm{s}$ injection), the imaging delay would be $12 \mathrm{~s}$ (i.e., start the scan $12 \mathrm{~s}$ after beginning the injection). Of course this times the bolus such that the midpoint arrives as central $\mathrm{k}$-space is collected, meaning that, depending on injection duration, there could already be venous enhancement by the time central k-space is collected. In this case, one may want to subtract a couple of seconds from the imaging delay time.

The most difficult aspect of the best guess technique is estimating the contrast travel time. As previously alluded to, it is better to image slightly late (increased venous phase) than slightly early (ringing/ banding artifact; Fig. 6B). With this in mind, as a general guideline for travel time from an antecubital vein to the abdominal aorta, we start with approximately $15 \mathrm{~s}$ for a young healthy patient. A young hypertensive or athletic patient would need fewer seconds. A healthy elderly patient would need approximately $20-25 \mathrm{~s}$. Patients with cardiac disease or an aortic aneurysm would be in the 25-35-s range. Severe cardiac failure in conjunction with aortic pathology might be up to $40-50 \mathrm{~s}$. We add 3-4 extra seconds if the intravenous line is in the hand.

\section{Test bolus technique}

A better, although somewhat more laborious way to estimate contrast travel time is through use of a test bolus $[69,70]$. With this technique, $1-2 \mathrm{cc}$ of gadolinium (followed by $10-15$-cc saline flush) are injected at the same rate as that planned for the actual injection. Multiple single-slice fast gradient echo images of the appropriate vascular region are then obtained as rapidly as possible (at least every 1-2 s) for approximately $1 \mathrm{~min}$. The time of peak arterial enhancement (contrast travel time) is then determined visually or by using region-of-interest analysis. Timing is then determined from equation 4 . To minimize time of flight effects, the 2D test bolus image should be either oriented along the vessel of interest (i.e., sagittal or coronal for the aorta) or relatively thick $(>1 \mathrm{~cm})$.

Using the test bolus technique for abdominal aortic imaging, Earls et al. demonstrated a mean aortic SNR increase of $902 \%$ (15 patients) following a $1 \mathrm{ml}$ bolus of gadopentetate dimeglumine [69]. By utilizing the resultant contrast travel time $(24.0 \pm 12.6 \mathrm{~s})$, they were $100 \%$ successful in obtaining a "pure" arterial phase study (as compared with $80 \%$ with best-guess timing) and saw significantly increased aortic SNR (29.8 vs. 20.5).

There are three main drawbacks to the test bolus technique. First, setting up, performing, and analyzing the test bolus lengthens the overall examination time. In the hands of Earls et al., the average time penalty was less than 5 min [69]. Second, the test bolus will rapidly redistribute into the interstitial space, thereby increasing background signal. For such small test doses, however, these effects are negligible. Finally, there is no absolute guarantee that the imaging bolus will behave identical to the test bolus because of moment-to-moment pa- tient variables such as venous return and cardiac output and the different total volume of injection.

\section{Automated bolus detection}

An automated (and hence simpler) yet somewhat more sophisticated approach for assuring proper bolus timing involves directly monitoring a vascular structure for arrival of contrast material and then "triggering" a centric acquisition once contrast arrival is detected (MR Smartprep, GE Medical Systems, Milwaukee, WI) [68, 71]. In this technique, signal from a large (approximately $10 \times 4 \times 4 \mathrm{~cm}$ ) "monitor" voxel overlapping the appropriate vascular structure (user defined, e.g., abdominal aorta) is rapidly obtained by using a simple spin echo sequence (time resolution $\sim 20 \mathrm{~ms}$ ). Initially, the sequence gathers approximately $20 \mathrm{~s}$ worth of data from the monitor voxel and calculates a baseline signal intensity and standard deviation. The sequence then continues to collect monitor data as contrast material is injected. Signal intensity is compared with the baseline in real time, and when it exceeds one or a combination of thresholds (typically a signal increase of greater than $15-20 \%$ and 3-4 SD above the mean), the sequence "triggers," meaning that it first pauses (user defined, typically $4-5 \mathrm{~s}$ ) to allow intravascular [Gd] to peak and breath-holding to initiate, then collects centric 3D SPGR data. This method ensures that peak arterial $[\mathrm{Gd}]$ and central k-space occur simultaneously.

Applying this automated technique to 50 consecutive patients undergoing abdominal MRA, Prince et al. found that for the same dose, arterial enhancement was $47 \%$ greater and inferior vena cava enhancement $57 \%$ less using the trigger when compared with 15 patients undergoing the best-guess technique (both values statistically significant) [68]. Alternatively, they found comparable aortic enhancement to best-guess timing by using $30 \%$ less gadolinium. Although they did have a $19 \%$ trigger "failure rate," this rate was mainly due to hardware/software problems in the early prototype sequence, which have been resolved.

The main pitfalls of this technique are improper placement of the tracker volume and patient movement such that the tracker volume is no longer aligned with the appropriate vascular structure. A user-defined default time is set to the maximum anticipated contrast travel time so that in the event of a trigger failure, a "salvage" study is obtained with delayed, but hopefully still diagnostic, bolus timing.

\section{MR fluoroscopy}

Another method of properly timing the contrast bolus still under development at this time makes use of extremely rapid "fluoroscopic", MR imaging [58, 72]. With this technique, a parallel array processor is employed to reconstruct rapidly the sagittal $2 \mathrm{D}$ gradient refocused images through the vascular structure of interest. In this fashion, images are generated in near real time and updated approximately once per second. The operator can watch the contrast bolus arrive and then switch over to a centric 3D imaging sequence when the desired enhancement is detected. Alternatively, automatic computer analysis can be performed, and the sequence can "trigger" in a fashion similar to the automated bolus detection.

This technique has the advantage of allowing real-time operatordependent decision making, which may be particularly advantageous in the evaluation of cases with unusual or asymmetric flow patterns, for optimizing venous signal, or in circumstances where it is difficult to choose a location for conventional triggering. As an example, enhancement downstream of a high-grade stenosis or complete vessel occlusion may be delayed compared with the normal side or may fill in later from collateral flow. Because such abnormalities may not be recognized beforehand, automated triggering may not provide optimal images. The ability to assess the vasculature "on the fly" under these circumstances could improve image quality. Although no commer- 
cially available MR scanners have this capability at present, adding the necessary hardware and software should not be problematic.

\section{Temporally resolved $3 D$ contrast MRA}

Because proper bolus timing is so difficult and a timing mistake can ruin the study, some investigators have advocated "temporally" resolved techniques whereby multiple 3D datasets are acquired (or synthesized) extremely rapidly (every $2-8$ s) [73-75]. With such techniques, bolus timing is no longer a factor because multiple vascular phases are obtained without any predetermined timing (i.e., inject and begin scanning simultaneously). The operator simply selects the desired image set, be it pure arterial, maximum venous, etc., which is particularly useful in the carotid arteries, where the venous phase is extremely rapid, and in the case of vessels with variable rate filling, as may occur with stenoses, occlusions, or AV fistulas [73].

Levy and Maki used a commercial imaging system to implement a "streamlined" 3D SPGR sequence that collected 12 slices with a temporal resolution of $7 \mathrm{~s}$ [73]. Using this system, they were able to differentiate reliably the arterial, early arteriovenous, late arteriovenous, and "washout" arteriovenous phases in the carotid arteries. As compared with standard breath-hold 3D MRA, their images suffered a considerable loss in volume coverage and SNR due to the small number of slices and partial Fourier techniques employed. Nonetheless, image quality was quite acceptable provided adequate vascular territory could be covered with the available number of slices.

Higher spatial resolution and increased volume coverage can be obtained by using the 3D TRICKS (time-resolved imaging of contrast kinetics) sequence $[74,75]$. In this technique, $\mathrm{k}$-space is divided into four blocks. The central block of k-space (which contributes most to image content) is repeatedly collected every $2-8 \mathrm{~s}$ in an alternating fashion with the other three blocks of more peripheral k-space. Images are then "synthesized" at 2-8-s temporal resolution by piecing together each unique central block of k-space with a linear interpolation of the remaining $\mathrm{k}$-space blocks acquired in closest temporal proximity.

This technique works well and allows greater temporal and spatial resolution/volume coverage than that obtained with a streamlined conventional sequence. The technique also lends itself to "video" format, where passage of contrast material can be viewed directly. The biggest drawback is the large amount of processing time required to perform the multiple reconstructions, which are currently done off-line. Also, $\mathrm{k}$-space discontinuities, in conjunction with changing intravascular gadolinium concentration, can potentially lead to artifacts. These artifacts, however, are small provided the gadolinium bolus is not too compact [76].

\section{Contrast-enhanced MRA: logistical considerations}

The following logistical considerations apply to 3D contrast MRA in general, whether using best-guess timing, a test bolus, or triggering.

\section{Artifacts \\ Related to $k$-space}

The key to maximizing arterial enhancement is to image such that the center of k-space is collected when arterial gadolinium concentration $[\mathrm{Gd}]$ is at a maximum. Acquiring central k-space data after arterial [Gd] has peaked leads to suboptimal arterial signal and increased venous signal (Figs. 5, 6C). Acquiring central k-space during the rapid upslope in arterial [Gd] causes "ringing"-type leading edge artifacts (Fig. 6B). Even with optimal timing, similar artifacts can be generated with the use of very compact boluses such that [Gd] falls rapidly while still acquiring relatively central k-space [76]. These artifacts are not as severe if $\mathrm{k}$-space is mapped in a sequential rather than a centric manner [57].

Thus, when using best-guess timing, sequential phase encoding is more forgiving. Also, our group tends to overestimate slightly the contrast travel time so that (inevitable) timing errors are more likely to cause increased venous enhancement than ringing artifacts. When using the test bolus technique with known contrast travel time, either centric or sequential phase encoding can be used. The advantage to centric encoding is twofold: less degradation with an incomplete breath-hold (see Respiratory section) and simplified operator logistics with respect to timing (no need to count backward to the middle of the scan to figure out when to inject; just inject, wait the proper delay, initiate breath-hold, and start imaging). For automated bolus detection (or fluoroscopic techniques), centric phase encoding is typically used. Because [Gd] rise times are not instantaneous, the delay from trigger to initiation of data acquisition becomes crucial to avoid artifacts. If the 3D MRA triggers at time $t_{1}$, the appropriate delay is $\left(t_{2}-t_{1}\right)$ (Fig. 5 ). This delay, of course, differs by patient and vascular territory. With trigger thresholds of $15-20 \%$ and 3-4 SD above the mean, a delay of 4-5 s worked well for abdominal aortic imaging [68]. In patients expected to have particularly slow flow (CHF, aneurysm), this time should be lengthened to 6-7 s. In the carotids, however, which have a more rapid upslope, trigger delays of $2-3 \mathrm{~s}$ are more appropriate to avoid jugular venous enhancement (work in progress).

\section{Respiratory}

Respiratory motion causes ghosting, blurring, and signal loss [77, 78]. In 3D imaging, blurring occurs in the direction of the motion, whereas ghosting is most pronounced in the slow phase encoding direction. Before the advent of fast imaging systems, 3D contrast MRA acquisition times were on the order of 3 min, making breath-holding an impossibility. Thus, early contrast MRA images suffered from significant image degradation, particularly when evaluating small vessels such as renal arteries [15]. Figure 7A,B illustrates the difference between breath-holding and free breathing.

Recently, many investigators have demonstrated the beneficial effects of breath-holding on 3D contrast MRA [13, 52-54, 62]. Holland et al. evaluated the same six patients using both breath-hold and nonbreath-hold 3D contrast MRA [13]. They found that the non-breathhold studies failed to identify three of four peripheral renal artery abnormalities, missed one of two accessory renal arteries, and were unable to visualize any of the renal artery bifurcations at the hilum. In contrast, breath-hold 3D contrast MRA identified all abnormalities, all accessory renal arteries, and renal artery bifurcations at the hilum in all patients.

Maki et al. looked at SNR loss and blur associated with incomplete breath-holds [62]. Their work demonstrated that breath-holding during the acquisition of the central $50 \%$ of $\mathrm{k}$-space was the most important for minimizing blur. Thus, for centric phase encoding, the majority of the benefit from breath-holding can be obtained even if the patient begins breathing at the midpoint of the scan. Data from a study by Wilman et al. shows that the more fully "centric" the phase encoding order is, the fewer the artifacts from partial breath-holds [61]. These data suggest that, for centric phase encoding orders, an optimistic assessment of a patient's breath-hold ability is in order because there is little to lose if they "don't quite make it."

Now that breath-hold 3D contrast MRA is a practical reality on most updated MR systems, breath-holding should be performed whenever possible. We have found that patients are better able to breathhold if someone explains the procedure and expectations well in advance of the actual scan. We find that the vast majority of ambulatory patients can breath-hold for at least $30 \mathrm{~s}$, and many have endurance well beyond this. Although performing a test breath-hold before the scan is always a possibility, watching a patient's respiratory pattern often provides a clue to the breath-hold potential. In our experience, patients who take slow deep breaths with relatively long expiratory pauses have no problem completing a 30-40-s breath-hold. Patients 


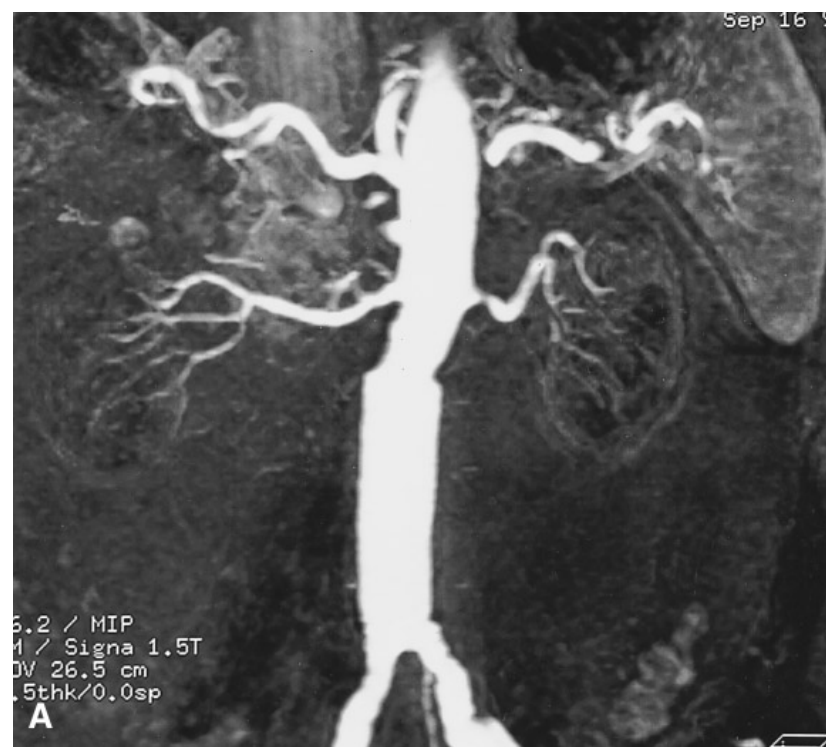

Fig. 7. MIP images from abdominal 3D gadolinium-enhanced MRAs (different patients). A Breath holding. B free breathing. Note the significant improvement in visualization of the distal renal arteries with

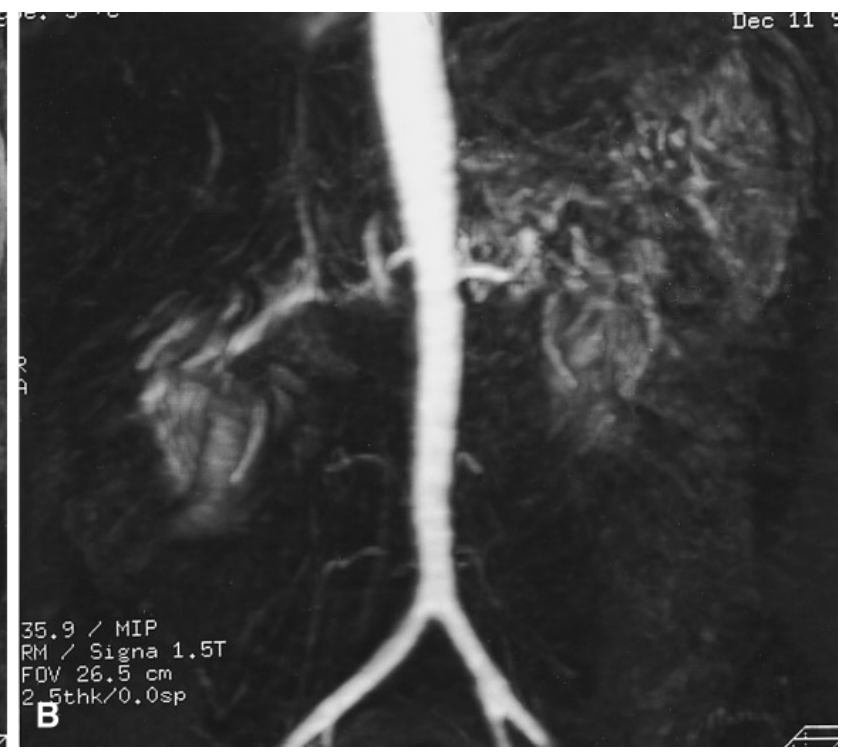

breath holding. Patient in A is shown after abdominal aortic aneurysm repair (from [62], with permission). who breath rapidly with no pause between expiration and subsequent inspiration often have trouble with a breath-hold of this duration. Sick or postoperative inpatients often cannot or will not hold their breath at all.

We recommend the following breathing strategy. For best-guess or test dose bolus timing techniques, pre-breath the patient for three or four cycles ("deep breath in . . . and breath out . . .") so that the last inspiration is held ("deep breath in and hold . . .") as the scan starts. Some investigators have advocated the use of supplemental oxygen during this pre-breathing [52]. For automated bolus detection or fluoroscopy techniques, breath the patient for three or four cycles and then have them relax and start the sequence to collect baseline data and wait for bolus arrival. Once the sequence triggers, use some prearranged signal such as tapping the arm to initiate a breath-hold. This signal needs to occur during the "delay" time, so that the patient is motionless once actual data collection starts.

\section{Determining imaging time}

The duration of a 3D contrast MRA acquisition ( $\mathrm{t}_{\mathrm{s}}$ ) depends on several variables and can be written as

$$
\mathrm{t}_{\mathrm{s}}=\text { YRES } * \text { ZRES } * \mathrm{TR}
$$

where YRES and ZRES are the number of phase encoding steps in y and $z$, and TR is the repetition time. TR is itself a function of echo time (TE), echo type (i.e., full or fractional), and bandwidth. Spatial resolution is determined by voxel size, which is determined by

voxel size $=(\mathrm{y}$ FOV/YRES $) \times(\mathrm{z}$ FOV/ZRES $)$

$$
\times(\mathrm{x} \mathrm{FOV/XRES})
$$

where $\mathrm{x}, \mathrm{y}$, and $\mathrm{z}$ FOV refer to the $\mathrm{x}, \mathrm{y}$, and $\mathrm{z}$ fields of view, and XRES is the number of data points digitized during readout. These parameters must be juggled to achieve the necessary volume coverage, spatial resolution, and SNR while keeping the scan time $t_{\mathrm{s}}$ within the anticipated breath-hold duration $\mathrm{t}_{\mathrm{b}}$ (or to at least no more than twice the breath-hold duration, per the argument of Maki et al. [62]). Thus, there are multiple trade-offs. Increasing resolution (YRES or ZRES with same overall slab thickness), increasing volume coverage (ZRES with same slice thickness), or increasing SNR (decreasing bandwidth or changing from fractional to full echo), increases scan time $\left(t_{s}\right)$. A mathematical analysis specific to breath-hold Gd-MRA for optimizing SNR by considering these variables is underway.

One method sometimes employed to reduce scan time without a loss in resolution is to use an asymmetric FOV (i.e., decrease both y FOV and YRES symmetrically). This method decreases scan time (while preserving resolution) at the expense of a smaller y FOV, which can be useful, particularly with large FOV studies where structures at the periphery of the y FOV are not important and wrap around (aliasing) is not an issue. Another useful technique without an associated time penalty is to zero-fill $\mathrm{k}$-space in the slice direction [79]. A $2 \times$ zero-fill yields twice the number of slices. Although the slice thickness is unchanged, the slices now overlap by one half a slice thickness. Although this technique does not actually increase resolution, it provides for smoother reformats by reducing partial volume effects.

Thus, the art of 3D breath-hold contrast MRA boils down to a careful choice of the parameters in equations 5-6 and is often an iterative process. As a rule, we start from the maximum estimated breath-hold duration $\left(\mathrm{t}_{\mathrm{b}}\right)$ and desired slice thickness. We then increase ZRES to cover the required volume. If an asymmetric FOV can be used, we do so. We then increase YRES until $t_{b}$ is reached (minimal value of 128). If $t_{s}$ cannot be made less than $t_{b}$ after this manipulation, then sacrifices must be made. Partial Fourier techniques can be used (i.e., collect only a certain fraction of y phase encodes) at the cost of decreased SNR. Alternatively, bandwidth can be increased, also at the cost of decreased SNR. Slice thickness can be increased at the cost of decreased spatial resolution. Finally, incomplete breath-holding can be anticipated at the cost of increased ghosting and blurring.

In cases where breath-holding is not as important, such as in the extremities or perhaps the carotids, there is more flexibility in choosing imaging parameters. In these circumstances, the limiting factor becomes contrast bolus duration. A "compact" bolus (with respect to imaging duration) causes distortion in a similar fashion to the ringing artifacts that occur when central $\mathrm{k}$-space is acquired during the leading edge of intravascular [Gd]. Thus, assuming a double dose $(0.2 \mathrm{mmol} /$ $\mathrm{kg})$ of a gadolinium chelate $(0.5 \mathrm{~mol} / \mathrm{L})$ for a $70-\mathrm{kg}$ patient adminis- 
tered at $2 \mathrm{cc} / \mathrm{s}$, the injection duration is only $14 \mathrm{~s}$. Assuming the bolus will broaden by several seconds before reaching the target vessels, this broadening is still less than approximately $20 \mathrm{~s}$. To avoid artifacts, this needs to be a substantial fraction of the scan time $t_{s}$, and scan times much greater than approximately $60 \mathrm{~s}$ are probably counterproductive. Nonetheless, this increase in imaging time allows for increased SNR and spatial resolution. When considering carotids, the rapidity of venous return also must be considered. This rapidity limits scan duration because the longer the scan, the closer the proximity of phase encoding to central k-space when venous signal arrives, which leads to greater venous signal and leading-edge venous artifact. We suspect carotid imaging durations of much greater than $30 \mathrm{~s}$ will significantly degrade image quality. As Wilman and Riederer pointed out, however, the more truly "centric" the phase encoding, the less degradation there will be from a compact bolus [60].

\section{Patient preparation}

The more relaxed and informed a patient is, the more easily that patient can remain still and perform a long breath-hold. Therefore, reassurance and a brief description of the scan can really help. In patients who are particularly anxious, premedication with sedatives such as diazepam may be useful. Premedication helps the patient to relax and lie still and also decreases cardiac output [14]. This latter effect helps enhance image quality because arterial phase $[\mathrm{Gd}]$ increases with decreasing cardiac output (equation 3, Fig. 3).

Because most 3D contrast MRA is performed in the coronal plane, arm position is important to allow for a smaller FOV without aliasing. For carotid, large FOV aortic, or runoff studies, the arms can remain by the patient's side because aliasing is not a factor. For pulmonary, small FOV aortic, renal, and mesenteric studies, the arms must be either elevated out of the imaging plane using cushions or extended over the head [52]. Both of these positions in which the arms are elevated have the added benefit of gravity-aided venous return.

We recommend placing the intravenous device before the patient is in the magnet. This procedure alleviates anxiety and possible shifts in patient position that may occur if the device is placed just before contrast injection. If the MRA will be performed with the arms by the patient's side, placing the intravenous device in the antecubital vein is adequate. If the arms will be over the head, it is preferable to place the device below the antecubital fossa so it will not kink and obstruct should the patient's elbow bend. A 22-gauge catheter is usually adequate and, if injecting by hand, will provide enough resistance that excessively rapid injections are not made. With gadolinium preparations such as gadopentetate dimeglumine (Magnevist, Berlex Laboratories, Wayne, NJ), however, warming to body temperature is required to decrease the viscosity enough to allow for adequate injection rates through a 22 -gauge intravenous device.

\section{Gadolinium injection}

The total gadolinium dose and rate of delivery are trade-offs between maximizing intravascular signal and minimizing artifacts. In the ideal situation, a large dose at a high rate would be optimal, but this, however, must be weighed against safety, practicality, and cost. We feel that for breath-hold contrast MRA with accurate bolus timing (test bolus technique, automated detection, fluoroscopy), a double dose ( 0.2 $\mathrm{mmol} / \mathrm{kg}$ ) administered at a rate of approximately $2 \mathrm{cc} / \mathrm{s}$ represents a good balance between these factors. In practice, however, once a bottle $(20 \mathrm{~mL})$ of gadolinium is opened, it is best to inject the entire bottle. Hence, we round the dose up to the next higher number of bottles, which generally amounts to two bottles $(40 \mathrm{~mL})$. For best-guess-type techniques, we prefer $0.3 \mathrm{mmol} / \mathrm{kg}$ at the same approximate rate. These recommendations can, under certain circumstances, be modified. For example, in a particularly small patient in whom the volume of a double dose is small, artifact from a compact bolus may be gen- erated. To remedy this result, either the injection rate can be decreased (thereby lengthening the bolus at the expense of intravascular signal) or the dose can be increased (at the expense of increased cost). If cost is an overwhelming concern, the dose and injection rate can be decreased at the expense of intravascular signal, or the dose alone can be decreased at the expense of compact bolus artifact.

In experienced hands, we find that manual injection works quite well. Because many institutions do not have a power injector, manual injection often is the only choice. Manual injections are also simpler in some ways because there is less "plumbing" and less opportunity for mechanical difficulties. That said, a power injector (e.g., Spectris MR Injector, MedRad, Pittsburgh, PA) offers a more constant and standardized bolus delivery. Either way, the contrast injection itself must be followed rapidly by adequate saline flush to complete delivery of the bolus and help flush the arm veins. Suggested flush volumes range from 15 to $50 \mathrm{cc}[52,69]$, with most investigators using $15-20$ cc. The rate of flush is usually the same as the gadolinium bolus itself. For hand injections, a standardized tubing set that allows rapid switchover between gadolinium and saline injection works best (Smartset, TopSpins, Plymouth, MI). Most power injectors automatically follow gadolinium delivery with a user-defined saline bolus, which eliminates possible delays in "switchover" with manual injection.

\section{Imaging different vascular phases}

With most 3D contrast MRA studies, extensive efforts are made to image the arterial phase optimally, as has been extensively discussed. Once the arterial phase dataset is collected, however, the sequence can (and should) be repeated to obtain venous and equilibrium phases. Later phases are useful in evaluating portal venous or venous structures, parenchymal enhancement patterns, and perhaps even renal glomerular function [80]. Temporal resolution is determined by a combination of scan time and the time required for the patient to prepare for another breath-hold. Aside from techniques such as 3D TRICKS, the faster the scan, the better the potential temporal resolution [75]. In cases where high temporal resolution is desired (such as perhaps in the carotids or in cases of AV shunts), the previously described streamlined techniques can be used in a single breath-hold, although SNR and spatial resolution decrease [73]. In more "conventional", breath-hold 3D contrast MRA, with a scan time of $\sim 30 \mathrm{~s}$, we advocate giving the patient approximately $10-15 \mathrm{~s}$ to "catch their breath" and then scan again. In this manner, the second "arteriovenous" phase occurs approximately $40-45 \mathrm{~s}$ after peak arterial phase. This is usually well timed for the portal venous phase and often adequate for evaluating venous structures and parenchymal phases in the visceral organs (Fig. 6C). Sometimes a third run may be desired, usually to evaluate slow venous return or the renal collecting system.

Because these "later" phase examinations occur more in the equilibrium phase of gadolinium distribution, intravascular $\mathrm{T} 1$ is increased (Fig. 2), and therefore signal is reduced as compared with arterial phase (Fig. 6A,C). To maximize signal in these later phases, the flip angle should, if possible, be reduced (see Fig. 1). We feel that whereas the optimum flip angle for arterial phase is $45-55^{\circ}$, the optimal flip angle decreases to $20-30^{\circ}$ in later phases.

\section{Postprocessing and display}

Three-dimensional Gd-MRA produces a contiguous volume of image data. For a typical body MRA study, this volume is asymmetric, being perhaps $32 \times 32 \times 6.4 \mathrm{~cm}$. For a representative acquisition matrix size of $256 \times 160 \times 32$, this volume produces voxels $1.25 \times 2.0 \times$ $2.0 \mathrm{~mm}$ in size. For diagnostic purposes, this dataset is best viewed interactively using a computer workstation allowing for thin multiplaner reformatting $[1,81]$. In this manner, thin $(1.25-2.0 \mathrm{~mm}$ in this case) slices can be viewed in axial, sagittal, coronal, or oblique planes, thus eliminating overlapping structures and unfolding tortuous ves- 

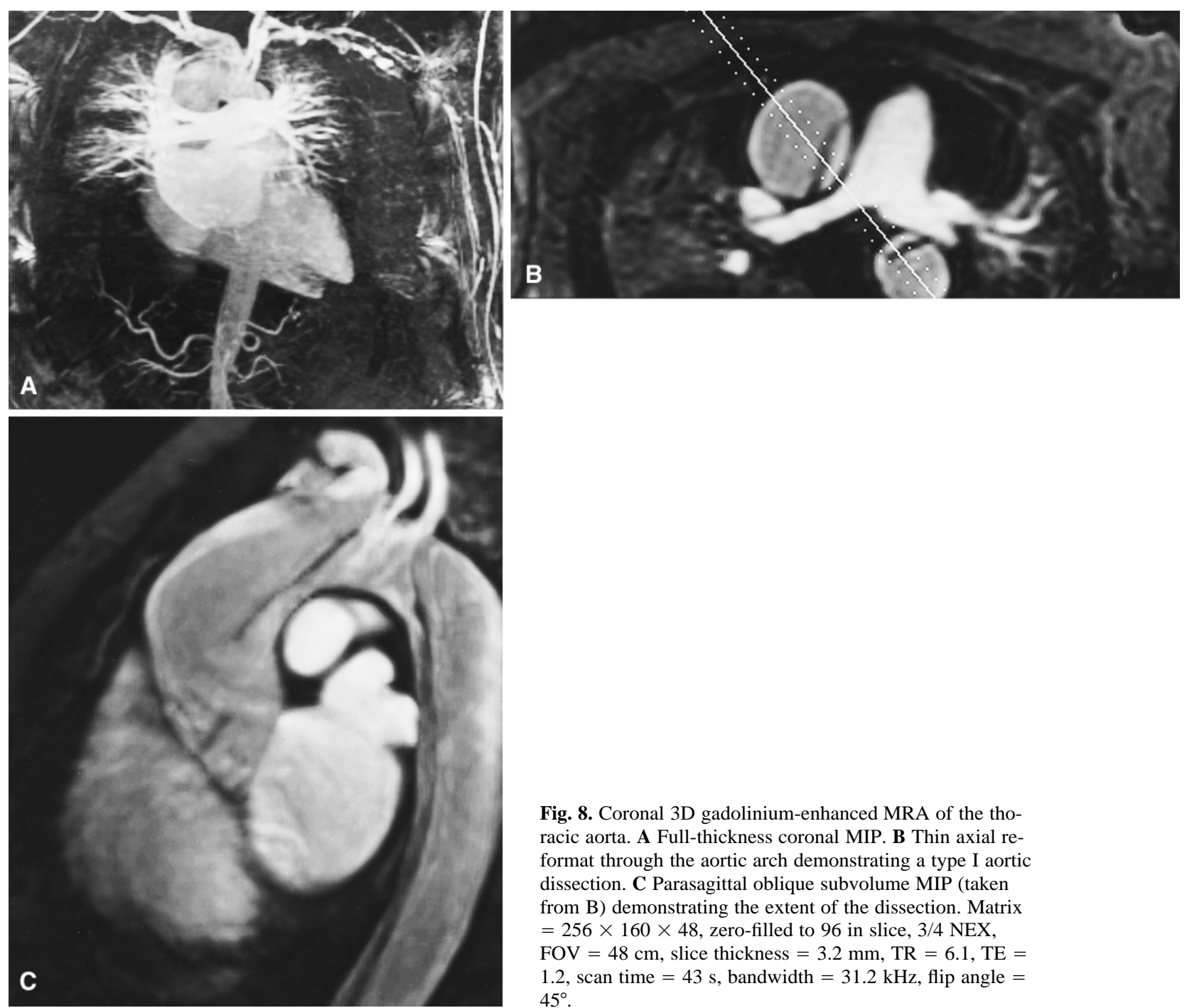

Fig. 8. Coronal 3D gadolinium-enhanced MRA of the thoracic aorta. A Full-thickness coronal MIP. B Thin axial reformat through the aortic arch demonstrating a type I aortic dissection. C Parasagittal oblique subvolume MIP (taken from B) demonstrating the extent of the dissection. Matrix $=256 \times 160 \times 48$, zero-filled to 96 in slice, $3 / 4 \mathrm{NEX}$, $\mathrm{FOV}=48 \mathrm{~cm}$, slice thickness $=3.2 \mathrm{~mm}, \mathrm{TR}=6.1$, TE $=$ 1.2 , scan time $=43 \mathrm{~s}$, bandwidth $=31.2 \mathrm{kHz}$, flip angle $=$ $45^{\circ}$.

sels. Because these reformatted slices remain thin and are not projections, they not only provide the best achievable contrast but also minimize the chance of diagnostic error when used diligently (see Fig. $8 \mathrm{~B})[1,81]$.

Because thin reformations show only short vascular segments, it is advantageous to utilize the maximum intensity projection (MIP) postprocessing technique [82, 83]. Using this algorithm, the user first specifies the subvolume thickness and desired viewing plane. The algorithm then generates rays perpendicular to the viewing plane, takes the maximum value of any voxel encountered along that ray, and assigns that maximum value to the corresponding pixel in the output image. This technique is fast and works extremely well with techniques such as Gd-MRA because vascular signal is typically much greater than background signal. It provides projection images that are quite similar to conventional angiograms in appearance. The MIP algorithm is demonstrated in Figure 8, which shows a full thickness coronal MIP of the thoracic aorta (Fig. 8A), a thin slice axial reformation through the superior aspect of the aortic arch (Fig. 8B), and an oblique subvolume MIP taken from the axial reformat demonstrating the origins of the great vessels and an extensive type I aortic dissection (Fig. 8C). As can be seen, this technique is very helpful for displaying complex vascular anatomy, particularly when vessels are not oriented along a single plane.
Despite its usefulness, the MIP algorithm is subject to artifacts. Perhaps the most common artifact arises when stationary tissue has greater signal intensity than the vascular structures, as can occur in the presence of fat, hemorrhage, metallic susceptibility artifacts, or motion artifacts. This in turn leads to the mapping of nonvascular signal to the projection image and causes a discontinuity in vessel signal, potentially mimicking stenosis or occlusion [1]. This type of artifact is best overcome by minimizing the thickness of the MIP subvolume, thereby excluding as much extraneous data as possible. Other artifacts inherent to the MIP technique have been described [84]. Such artifacts mainly consist of underestimating vessel lumen and are more of a problem with TOF or PC techniques. Because of these potential pitfalls, most investigators agree that the MIP images should be used as a roadmap and use the source images for definitive diagnosis [1, $81,84]$.

Subtraction techniques are also useful in image evaluation, particularly in vascular regions not subjected to significant respiratory motion. These areas include the extremities, pelvis, and carotids. A "digital subtraction" MRA can be accomplished most easily by subtracting pre- from postcontrast magnitude (reconstructed) images. Provided the patient maintains the same position on both studies, subtraction will eliminate background signal and improve vessel conspicuity. This technique has been used successfully in the extremities 


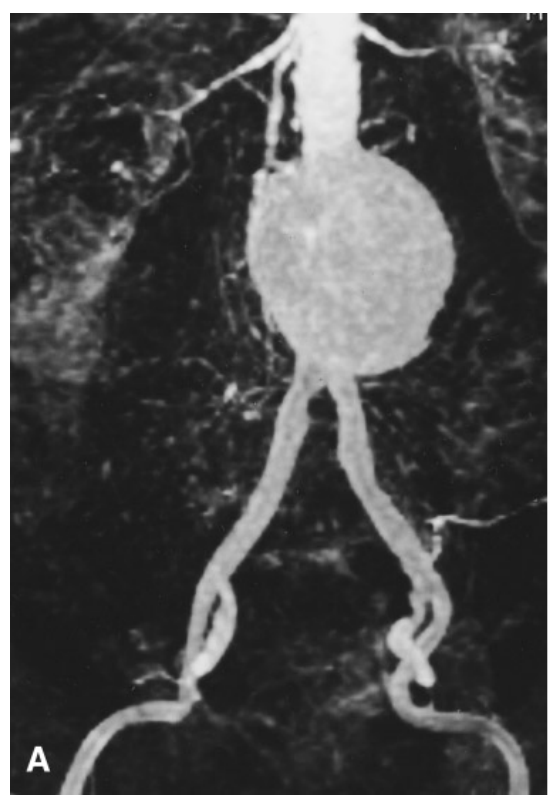

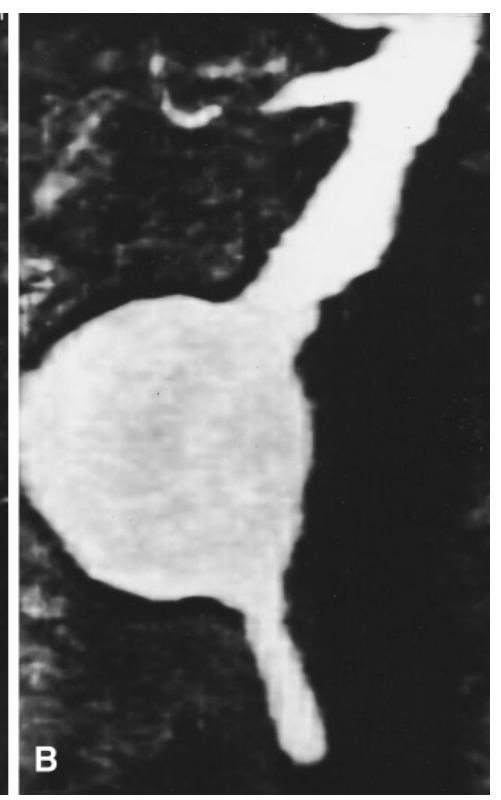

Fig. 9. Coronal (A) and sagittal (B) MIP reformats from a 3D gadolinium-enhanced MRA of the abdominal aorta demonstrating a large infrarenal saccular aneurysm. The renal, celiac, superior mesenteric, and iliac arteries are normal. Automated bolus detection with breath-hold. Matrix $=256 \times$ $160 \times 48, \mathrm{FOV}=32 \mathrm{~cm}$, slice thickness $=2.7$ $\mathrm{mm}, \mathrm{TR}=6.5$, TE $=1.8$, scan time $=51 \mathrm{~s}$, bandwidth $=31.3 \mathrm{kHz}$, flip angle $=45^{\circ}$.
$[85,86]$. An improvement on this technique involves complex subtraction of the pre- and postcontrast "raw" (k-space) datasets, which overcomes phase differences in voxels that contain both stationary and moving spins (i.e., very small vessels), allowing for increased vascular signal [87].

\section{Contrast-enhanced MRA: clinical applications in the abdomen}

This section reviews techniques and imaging parameters for gadolinium-enhanced MRA in the abdomen. The parameters provided here were derived from clinical experience with the Horizon 1.5-T system (GE Medical Systems, Milwaukee, WI) using software version 5.5 or 5.6 with echo planer capable gradients (SR110) and the body coil. The general techniques described in the present section are applicable to other systems and vascular territories, although the parameters may need to be extrapolated as appropriate.

Based on the previous discussion, several general principles apply when performing Gd-MRA. First, TR and TE need to be as short as possible without excessively increasing the bandwidth. Echo time (TE) can be minimized by using a fractional (asymmetric) echo. For the Horizon system using a bandwidth of $\pm 32 \mathrm{kHz}$, minimum TR/TEs of approximately 6.0-7.0/1.2-2.0 $\mathrm{ms}$ are possible. Other systems are capable of an even shorter TR. Second, slice thickness should be no greater than $2.0-2.6 \mathrm{~mm}$, unless imaging large structures where fine detail is not of great concern (such as the portal vein), in which case slice thicknesses of up to 3.0-3.5 $\mathrm{mm}$ are often acceptable. Third, in cases where breathholding will be performed (advantageous everywhere, essential in the abdomen), the number of slices and phase encoding steps must be balanced among adequate volume coverage, adequate resolution, and constrained breath-holding time. For a typical FOV of $32 \mathrm{~cm}, 128$ phase encodings (the minimum number usually used) corresponds to a resolution of $2.5 \mathrm{~mm}$. For 128 phase encodings and a TR of $7 \mathrm{~ms}$, approximately 44 slices can be obtained in a 40-s breath-hold or 22 slices in a 20 -s breath-hold. These imaging times can be reduced further by using partial Fourier imaging (1/2 NEX). Finally, for most arterial phase studies, it is best to use an automated bolus detection technique, which is particularly true for renal and carotid studies, where too much imaging delay causes interference due to adjacent venous opacification. If automated bolus detection is not available, a test bolus can be used. If neither of these techniques are feasible, best-guess timing can be undertaken.

\section{Abdominal aorta}

Three-dimensional gadolinium-enhanced MRA of the abdominal aorta is performed most often to evaluate dissection (Fig. 8) or aneurysmal disease (Fig. 9). The GdMRA sequence is typically performed in a coronal plane (so that the iliac and renal vessels also can be evaluated) and is prescribed from a 2D "black blood" gradient refocused breath-hold sagittal localizer. Prescribing from a breath-hold localizer serves several purposes. First, it is fast. Second, it captures the anatomy in the same breath-hold position in which the Gd-MRA will performed, thus decreasing the chances of having a vital structure (such as the anterior aspect of an aneurysm) fall outside the FOV. Finally, it gives the patient a chance to practice a breath-hold when the outcome is not crucial. 

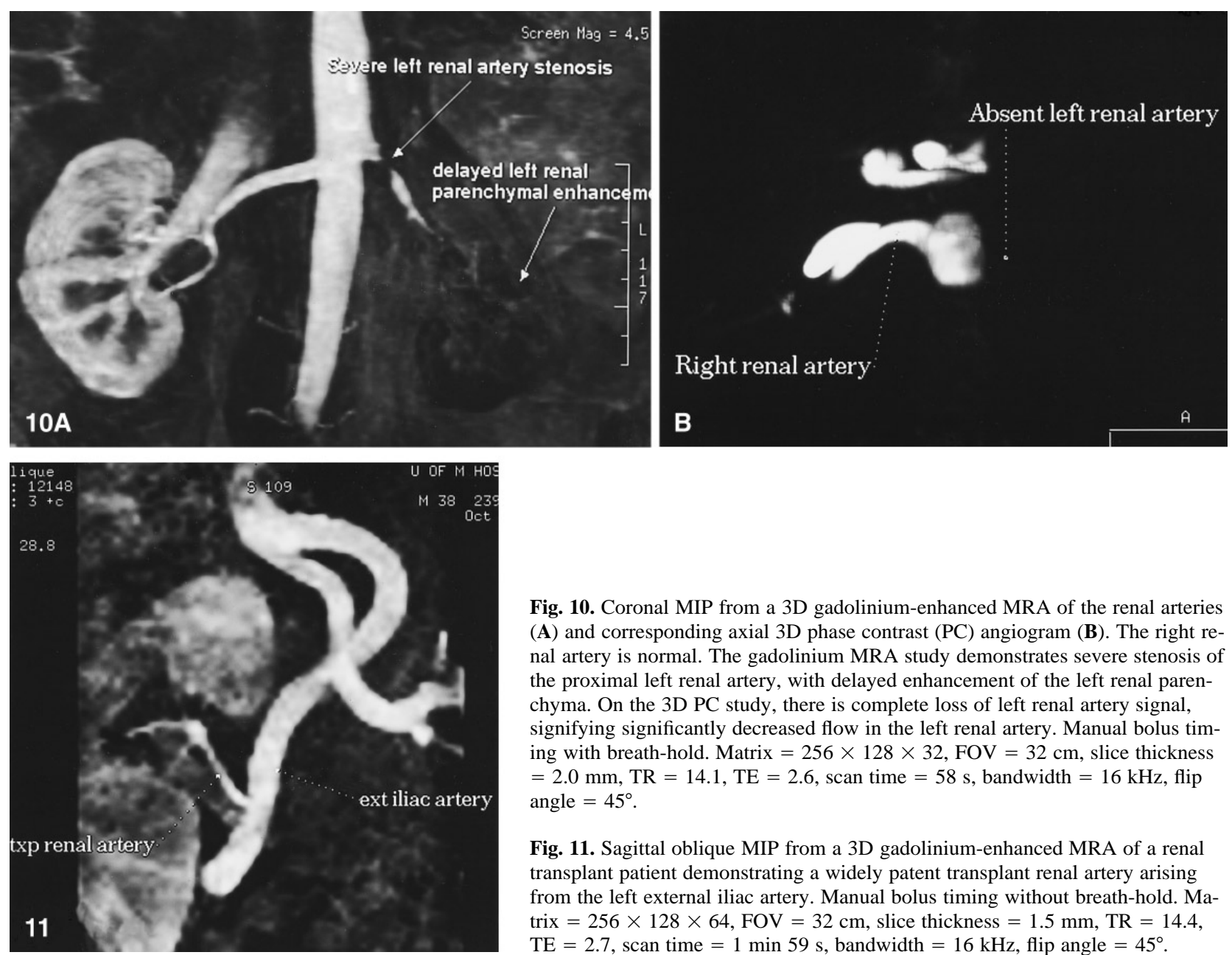

Fig. 10. Coronal MIP from a 3D gadolinium-enhanced MRA of the renal arteries (A) and corresponding axial 3D phase contrast (PC) angiogram (B). The right renal artery is normal. The gadolinium MRA study demonstrates severe stenosis of the proximal left renal artery, with delayed enhancement of the left renal parenchyma. On the 3D PC study, there is complete loss of left renal artery signal, signifying significantly decreased flow in the left renal artery. Manual bolus timing with breath-hold. Matrix $=256 \times 128 \times 32$, FOV $=32 \mathrm{~cm}$, slice thickness $=2.0 \mathrm{~mm}, \mathrm{TR}=14.1, \mathrm{TE}=2.6$, scan time $=58 \mathrm{~s}$, bandwidth $=16 \mathrm{kHz}$, flip angle $=45^{\circ}$.

Fig. 11. Sagittal oblique MIP from a 3D gadolinium-enhanced MRA of a renal transplant patient demonstrating a widely patent transplant renal artery arising from the left external iliac artery. Manual bolus timing without breath-hold. Matrix $=256 \times 128 \times 64, \mathrm{FOV}=32 \mathrm{~cm}$, slice thickness $=1.5 \mathrm{~mm}, \mathrm{TR}=14.4$ $\mathrm{TE}=2.7$, scan time $=1 \min 59 \mathrm{~s}$, bandwidth $=16 \mathrm{kHz}$, flip angle $=45^{\circ}$.

A 32-cm FOV is usually adequate for Gd-MRA of the abdominal aorta. If the patient is very large or has iliac/thoracic aortic disease, the FOV can be increased as needed. As the FOV increases, however, resolution decreases, and evaluating the renal arteries becomes more difficult. Slice thickness and number of slices are chosen based on desired resolution and breath-holding capability. Ideally, slice thickness should be $\sim 2.5 \mathrm{~mm}$ or less. For very large aneurysms, however, it may be necessary to increase slice thickness to $3.0-3.5 \mathrm{~mm}$.

Because large aneurysms have slow, swirling flow, extra time is required for the aneurysm to opacify maximally. When using automated bolus detection, we find that a 5-s delay is optimal for a nondiseased aorta and that $7 \mathrm{~s}$ are more appropriate for patients with the exceedingly slow flow associated with a large aortic aneurysm or congestive heart failure. Extremely large aneurysms also may require a larger gadolinium dose; we often use three bottles $(60 \mathrm{~mL})$ under these circumstances.

Another factor worth considering when using automated bolus detection is monitor volume placement because many aneurysms (or false lumens in the case of dissection) are partially thrombosed. Thrombus often can be hard to distinguish from the sagittal localizer. Axial T1 or T2 images can help in localizing the patent portion of the lumen.

\section{Renal arteries}

Gadolinium-enhanced MRA of the renal arteries is performed similarly to the abdominal aortic study, except the FOV is kept to a maximum of $32 \mathrm{~cm}$ (minimum determined by patient size to avoid aliasing). Typically, 32 slices are obtained with a slice thickness no greater than $\sim 2.6 \mathrm{~mm}$. This thickness allows for high resolution with relatively short scan times $(<30 \mathrm{~s})$. Breath-holding is extremely critical because distal branches and accessory renal arteries are difficult to evaluate in the presence of respiratory motion. If the patient is a good breath-holder, resolution can be increased by decreasing slice thickness and increasing the number of phase encoding steps.

Figure 10A shows a severe proximal left renal artery stenosis. Three-dimensional PC imaging (Fig. 10B) can 

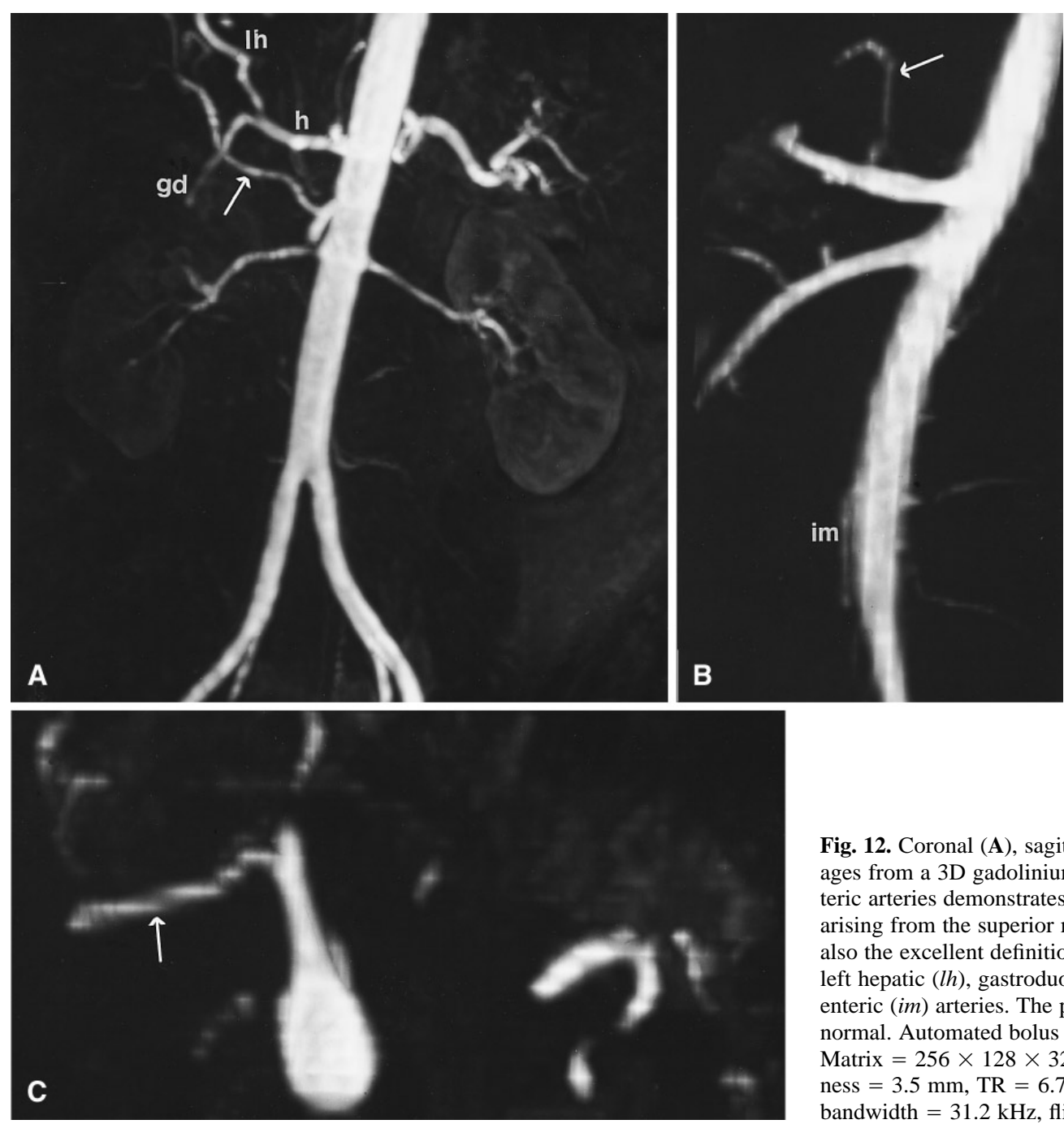

Fig. 12. Coronal (A), sagittal (B), and axial (C) MIP images from a 3D gadolinium-enhanced MRA of mesenteric arteries demonstrates a replaced right hepatic artery arising from the superior mesenteric artery (arrow). Note also the excellent definition of the common hepatic $(h)$, left hepatic $(l h)$, gastroduodenal $(g d)$, and inferior mesenteric (im) arteries. The proximal SMA and celiac are normal. Automated bolus detection with breath-hold. Matrix $=256 \times 128 \times 32$, FOV $=36 \mathrm{~cm}$, slice thickness $=3.5 \mathrm{~mm}, \mathrm{TR}=6.7, \mathrm{TE}=1.8$, scan time $=28 \mathrm{~s}$, bandwidth $=31.2 \mathrm{kHz}$, flip angle $=45^{\circ}$.

be a useful adjunct in the evaluation of renal artery stenosis. The Gd-MRA images demonstrate the anatomy, and the PC images show signal drop-out in regions of turbulence or extremely slow flow, which indicates the stenosis is hemodynamically significant [88].

Transplant renal arteries also can be evaluated (Fig. 11) [89]. Because these arteries are in the pelvis, respiratory motion is not as pronounced, and breath-holding is not as critical. Nonetheless, it is best to have the patient breath-hold if possible. Although coronal imaging with a $32-\mathrm{cm}$ FOV is usually adequate, sagittal imaging can be performed with a smaller FOV. This technique provides higher resolution but covers much less vascular territory. Transplant renal veins are easily seen on the venous phase of the study.

\section{Mesenteric arteries}

The mesenteric vessels are included on renal or abdominal aortic Gd-MRA studies (Fig. 12). The proximal ce- liac and superior mesenteric arteries are usually well visualized, even in the presence of respiratory motion. The inferior mesenteric artery is often seen.

Dedicated mesenteric artery studies are performed in the coronal plane. Slice thickness must be minimized $(<2.6 \mathrm{~mm})$ because the proximal mesenteric arteries travel primarily in the slice direction (note the somewhat "jagged" appearance of the 3.5-mm slices shown in Fig. 12B). Zero-filling in the slice direction helps to smooth out the sagittal reconstructions. Keep in mind that the hepatic, gastroduodenal, and distal superior mesenteric arteries may be farther anterior than is usually included in a renal study, and therefore volume coverage will need to be increased (or shifted anteriorly) to see these structures.

\section{Portal/hepatic veins}

Portal venous or hepatic vein studies are performed in a coronal plane (Figs. 13, 14). Slice thickness is gen- 

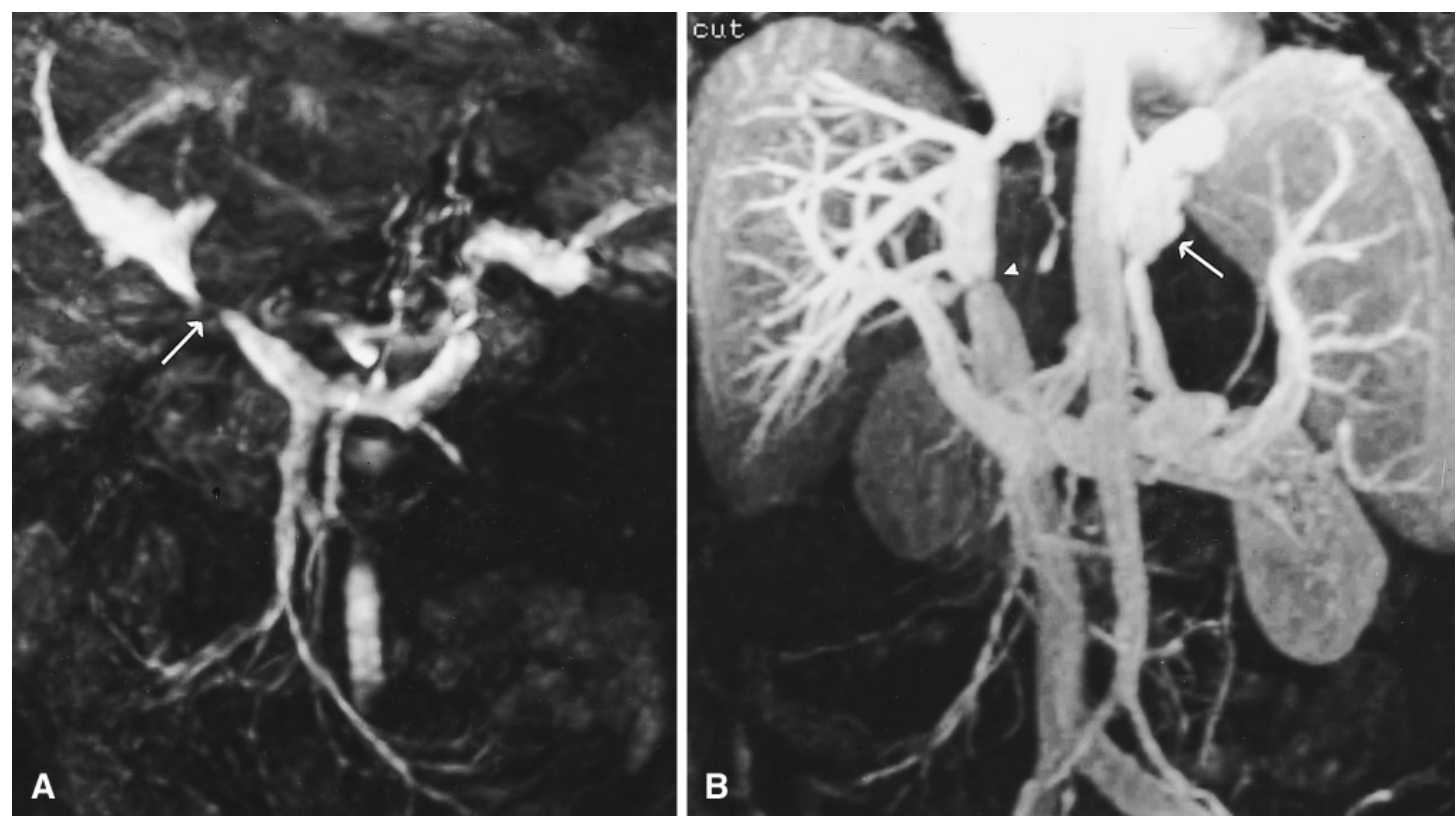

Fig. 13. Coronal MIP images from 3D gadolinium-enhanced MRA of the portal vein in two different patients after liver transplant. A Severe stenosis of the main portal vein (arrow) at the site of the anastamosis. The superior mesenteric and splenic veins are patent. Matrix $=256$ $\times 128 \times 32, \mathrm{FOV}=36 \mathrm{~cm}$, slice thickness $=3.0 \mathrm{~mm}, \mathrm{TR}=6.7, \mathrm{TE}$ $=1.8$, scan time $=28 \mathrm{~s}$, bandwidth $=32 \mathrm{kHz}$, flip angle $=45^{\circ} . \mathbf{B}$ Dilated gastroesophageal varices (arrow). Note also the mild stricture

of the infrahepatic IVC related to transplant surgery (arrowhead). Matrix $=256 \times 128 \times 32$ zero filled to 64 in slice, $3 / 4$ NEX, FOV $=48 \mathrm{~cm}$, slice thickness $=2.8 \mathrm{~mm}, \mathrm{TR}=7.0, \mathrm{TE}=2.1$, scan time $=18 \mathrm{~s}$, bandwidth $=32 \mathrm{kHz}$, flip angle $=45^{\circ}$. Both studies were obtained in the portal venous phase (approximately $60 \mathrm{~s}$ postinjection) with breath-hold.

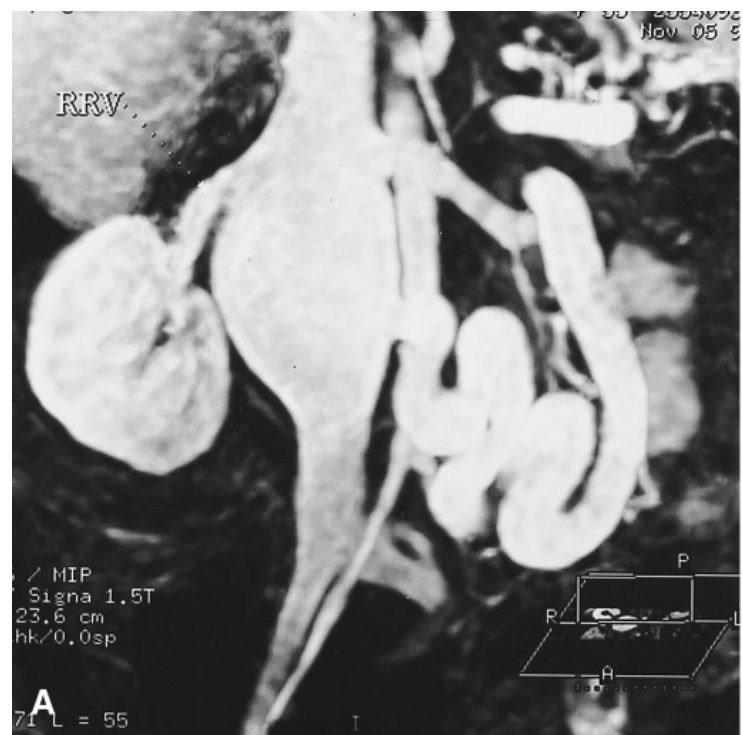

Fig. 14. Coronal (A) and oblique (B) MIP images from 3D gadolinium-enhanced MRA of the portal vein in a patient with severe portal hypertension after liver transplant. This case demonstrates a large inferior mesenteric venous collateral decompressing the portal system into the IVC, which is focally aneurysmal. Matrix $=256 \times 128$

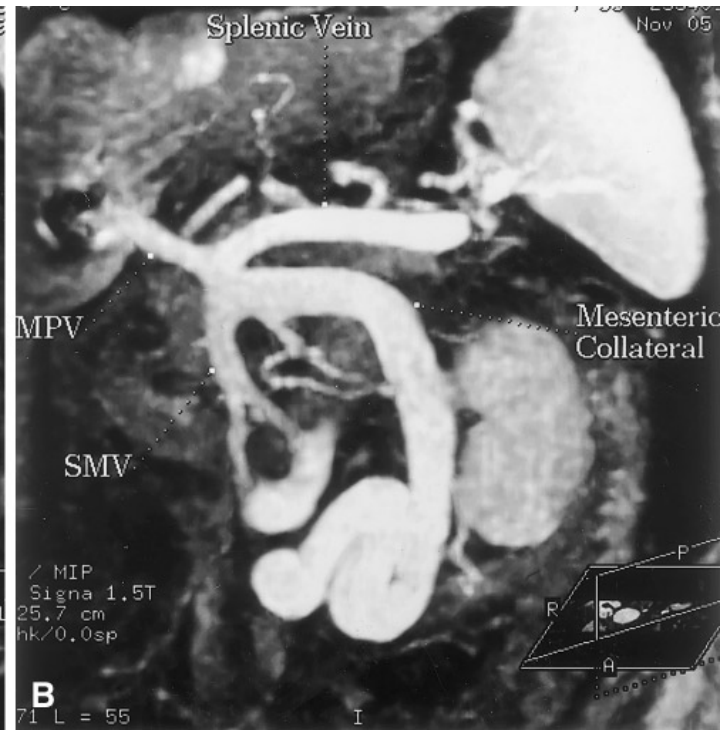

$\times 32, \mathrm{FOV}=36 \mathrm{~cm}$, slice thickness $=4.0 \mathrm{~mm}, \mathrm{TR}=5.1, \mathrm{TE}=1.2$, scan time $=21 \mathrm{~s}$, bandwidth $=31.2 \mathrm{kHz}$, flip angle $=45^{\circ}$. The study was obtained in the portal venous phase (approximately $60 \mathrm{~s}$ postinjection) with breath-holding. 
erally increased to $3.0-4.0 \mathrm{~mm}$ to adequately cover the relevant portal venous structures. An axial study is useful in determining the anterior and posterior extents of the portal/hepatic veins.

In portal venous/hepatic venous studies, timing is not as crucial as with arterial studies because the portal venous phase is longer and thus easier to isolate. After obtaining the arterial phase images, an approximately 5-10-s pause allows the patient to recover from the breath-hold. Imaging is then repeated, followed by a second pause and a third scan. In this manner, the second set of images usually provides a nice portal venous and hepatic venous phase. The third set may show collaterals to better advantage, if they exist.

This protocol has the added advantage of being essentially a dynamic liver study. Hence, if there is concern over a hepatic mass, this mass can be evaluated dynamically, provided it is included in the imaging volume. Under these circumstances, further delayed phases should be obtained for $10-15 \mathrm{~min}$.

\section{Summary}

Gadolinium-enhanced MRA in the abdomen provides a safe, fast, and cost-efficient alternative to conventional diagnostic angiography. Numerous recent advances allow for high-resolution breath-hold imaging with optimal arterial-phase gadolinium bolus timing. As the technique is refined further, greater spatial and temporal resolution will become possible, thus increasing the usefulness of Gd-MRA.

\section{References}

1. Saloner D. MRA: principles and display. In: Higgins CB, Hricak $\mathrm{H}$, Helms CA, eds. Magnetic resonance imaging of the body. Philadelphia: Lippencott-Raven Publishers, 1997;1345-1368

2. Gullberg GT, Wehrli FW, Shimakawa A, et al. MR vascular imaging with a fast gradient refocusing pulse sequence and reformattied images from transaxial sections. Radiology 1987; 165:241-246

3. Dumoulin CL, Cline HE, Souza SP, et al. Three-dimensional time-of-flight magnetic resonance angiography using spin saturation. Magn Reson Med 1989;11:35-46

4. Owen R, Baum RA, Carpenter JP, et al. Symptomatic peripheral vascular disease: selection of imaging parameters and clinical evaluation with MR angiography. Radiology 1993; 187:627-635

5. Cortell ED, Kaufman JA, Geller SC, et al. MR angiography of the tibial runoff vessels: imaging with the head coil compared to conventional arteriography. AJR 1996;167:147-151

6. Glickerman DJ, Obregon RG, Schmiedl UP, et al. Caridac gated MR angiography of the entire lower extremity: a prospective comparison with conventional arteriography. AJR 1996; 167:445-451

7. Patel MR, Kuntz KM, Klufas RA, et al. Preoperative assessment of the carotid bifurcation. Can magnetic resonance angiography and duplex ultrasonography replace contrast arteriography? Stroke 1995;26:1753-1758
8. Anderson CM. MRA of the aortic arch and extracranial carotid arteries. In: Higgins CB, Hricak H, Helms CA, eds. Magnetic resonance imaging of the body. Philadelphia: Lippencott-Raven Publishers, 1997;1369-1381

9. Blatter DD, Parker DL, Robinson RO. Cerebral MR angiography with multiple overlapping thin slab acquisition. Radiology 1991; 179:805-811

10. Kim D, Edelman RR, Kent KC, et al. Abdominal aorta and renal artery stenosis: evaluation with MR angiography. Radiology 1990; 174:727-731

11. Yucel EK, Kaufman JA, Prince MR, et al. Time-of-flight renal MR arteriography: utility in patients with renal insufficiency. Magn Reson Imaging 1993; 11:925-930

12. Loubeyre P, Cahen R, Grozel F, et al. Transplant renal artery stenosis. Evaluation of diagnosis with magnetic resonance angiography compared with color duplex sonography and arteriography. Transplantation 1996;62(4):446-450

13. Holland GA, Dougherty L, Carpenter JP, et al. Breath-hold ultrafast three-dimensional gadolinium-enhanced MR angiography of the aorta and the renal and other visceral abdominal arteries. AJR 1996; 166:971-981

14. Prince MR. Body MR angiography with gadolinium contrast agents. MRI Clin North Am 1996;4(1):11-24

15. Prince MR. Gadolinium-enhanced MR aortography. Radiology 1994; 191:155-164

16. Bryant D, Payne J, Firmin D, et al. Measurement of flow with NMR imaging using a gradient pulse and phase difference technique. J Comput Assist Tomogr 1984; 8:588-593

17. Gedroyc WMW. Magnetic resonance angiography of renal arteries. Urol Clin North Am 1994;21:201-214

18. Gedroyc WM, Negus R, Al-Kutoubi A, et al. Magnetic resonance angiography of renal transplants. Lancet 1992;339:789-791

19. Bass JC, Prince MR, Londy FJ, et al. Effect of gadolinium on phase-contrast MR angiography of the renal arteries. AJR 1997; 168:261-266

20. Yucel EK. Magnetic resonance angiography of the lower extremity and renal arteries. Sem Ultrasound CT MRI 1992;13(4):291-302

21. Ngheim HV, Winter TC, Mountford MC, et al. Evaluation of the portal venous system before liver transplantation: value of phasecontrast MR angiography. AJR 1995; 164:871-878

22. Lundin B, Cooper TG, Meyer RA, et al. Measurement of total and unilateral renal blood flow by oblique-angle velocity encoded 2D-CINE magnetic resonance angiography. Magn Reson Imaging 1992; 11:51-59

23. Schoenberg S, Knopp MV, Bock M, et al. MR flow measurements for assessment of different degrees of renal artery stenosis. Fourth scientific meeting and exhibition. New York: International Society for Magnetic Resonance in Medicine, 1996;164

24. Weinmann HJ, Brasch RC, Press WR, et al. Characteristics of gadolinium-DTAP complex: a potential NMR contrast agent. AJR 1984; 142:619-624

25. Schima W, Mukerjee A, Saini S. Contrast-enhanced MR imaging. Clin Radiol 1996;51(4):235-244

26. Goldstein HA, Kashanian FK, Blumetti RF, et al. Safety assessment of gadopentetate dimeglumine in U.S. clinical trials. Radiology 1990; $174: 17-23$

27. Niendorf H, Haustein J, Alhassan A, et al. Safety of gadoliniumDTPA: extended clinical experience. In: Brasch R, ed. Workshop on contrast-enhanced magnetic resonance. Napa, CA: Society for Magnetic Resonance in Medicine, 1991:70-79

28. Haustein J, Niendorf HP, Krestin G, et al. Renal tolerance of gadolinium-DTAP/dimeglumine in patients with chronic renal failure. Invest Radiol 1992;27:153-156

29. Prince MR, Arnoldus C, Frisoli JK. Nephrotoxicity of high dose gadolinium compared to iodinated contrast. JMRI 1996;6:162166

30. Rofsky NM, Weinreb JC, Bosniak MA, et al. Renal lesion characterization with gadolinium-enhanced MR imaging: efficacy and 
safety in patients with renal insufficiency. Radiology 1991; 180:85-89

31. Hohenschuh E, Watson AD. Contrast media: theory and mechanisms of contrast-enhancing agents. In: Higgins CB, Hricak H, Helms CA, eds. Magnetic resonance imaging of the body. Philadelphia: Lippencott-Raven Publishers, 1997:1439-1464

32. Schmiedl U, Brasch RC, Ogan MD, et al. Albumin labeled with Gd-DTPA. Acta Radiol 1990;374(suppl):99-102

33. Marchal G, Bosmans H, Van Hecke P, et al. MR angiography with gadopentetate dimeglumine-polylysine: evaluation in rabbits. AJR 1990; $155: 407-411$

34. Bogdanov AA, Weissleder R, Frank HW, et al. A new macromolecule as a contrast agent for MR angiography: preparation, properties, and animal studies. Radiology 1993;187:701-706

35. Loubeyre P, Canet E, Zhao S, et al. Carboxymethyl-dextran-gadolinium-DTPA as a blood-pool contrast agent for magnetic resonance angiography. Experimental study in rabbits. Invest Radiol 1996; 31:288-293

36. Saini S, Edelman RR, Sharma P, et al. Blood-pool MR contrast material for detection and characterization of focal hepatic lesions: initial clinical experience with ultrasmall superparamagnetic iron oxide (AMI-227). AJR 1995; 164:1147-1152

37. Anzai Y, Prince MR, Chenevert TL, et al. MR angiography with and ultrasmall superparamagnetic iron oxide blood pool agent. JMRI 1997; 7:209-214

38. Brasch RC. New directions in the development of MR imaging contrast media. Radiology 1992;183:1-11

39. Mayo-Smith WW, Saini S, Slater G, et al. MR contrast material for vascular enhancement: value of superparamagnetic iron oxide. AJR 1996; 166:73-77

40. Benderbous S, Bonnemain B. Supermagnetic nanoparticles as blood-pool contrast agents. Radiologe 1995;35(suppl 2):S248S252

41. Schmiedl U, Sievers RE, Brasch RC, et al. Acute myocardial ischemia and repurfusion: MR imaging with albumin-Gd-DTPA. Radiology 1989; 170:351-356

42. Saeed M, Wendland M, Masui, et al. Myocardial infarction: assessment with an intravascular MR contrast medium. Work in progress. Radiology 1991;180:153-160

43. Berthezene Y, Vexler V, Jerome H, et al. Differentiation of capillary leak and hydrostatic pulmonary edema with a macromolecular MR imaging contrast agent. Radiology 1991;181:773-777

44. Baxter AB, Melnikoff S, Stites DP, et al. Immunogenicity of gadolinium-based contrast agents for magnetic resonance imaging: induction and characterization of antibodies in animals. Invest Radiol 1991;26:1035-1040

45. Van Hecke P, Marchal G, Bosmans H, et al. NMR imaging study of the pharmacodynamics of polylysine-gadolinium-DTPA in the rabbit and the rat. Magn Reson Imaging 1991;9:313-321

46. McLachlan SJ, Morris MR, Lucas MA, et al. Phase I clinical evaluation of a new iron oxide MR contrast agent. JMRI 1994; 4:301-307

47. Prince MR, Yucel EK, Kaufman JA, et al. Dynamic gadoliniumenhanced three-dimensional abdominal MR arterography. JMRI 1993; 3:877-881

48. Runge VM, Kirsch JE, Lee C. Contrast-enhance MR angiography. JMRI 1993; 3:233-239

49. Ehman RL, Revel D, Sievers R, et al. Acute myocardial ischemia. Magnetic resonance contrast enhancement with gadoliniumDTPA. Radiology 1984; 153:157-163

50. Moseley ME, Sawyer AM. Imgaing techniques: pulse sequences. In: Higgins CB, Hricak H, Helms CA, eds. Magnetic resonance imaging of the body. Philadelphia: Lippencott-Raven Publishers, 1997:43-69

51. Wehrli FW. Principles of magnetic resonance. In: Stark DD, Bradley WG, eds. Magnetic resonance imaging. St Louis: MosbyYear Book Publishers, 1992:3-20

52. Prince MR, Narasimham DL, Stanley JC, et al. Breath-hold gadolinium-enhanced MR angiography of the abdominal aorta and its major branches. Radiology 1995; 197:785-792
53. Snidow JJ, Johnson MS, Harris VJ, et al. Three-dimensional gadolinium-enhanced MR angiography for aortoiliac inflow assessment plus renal artery screening in a single breath hold. Radiology 1996; 198:725-732

54. Leung DA, McKinnon GC, Davis CP, et al. Breath-hold, contrastenhanced, three-dimensional MR angiography. Radiology 1996;201:569-571

55. Hendrick RE, Roff U. Image contrast and noise. In: Stark DD, Bradley WG, eds. Magnetic resonance imaging. Chicago, IL: Mosby-Year Book, 1991;135

56. Weinmann HJ, Laniado M, Mützel W. Pharmacokinetics of GdDTPA/dimeglumine after intravenous injection into healthy volunteers. Phsyiol Chem Phys Med NMR 1984; 16:167-172

57. Maki JH, Prince MR, Londy FJ, et al. The effects of time varying intravascular signal intensity on three-dimensional MR angiography image quality. JMRI 1996;6:642-651

58. Riederer SJ, Tasciyan T, Farzaneh F. MR flouroscopy: technical feasibility. Magn Reson Med 1988;8:1-15

59. Bampton AEH, Riederer SJ, Korin HW. Centric phase-encoding order in three-dimensional MP-RAGE sequences: application to abdominal imaging. JMRI 1992;2:327-334

60. Wilman AH, Riederer SJ. Improved centric phase encoding orders for three-dimensional magnetization-prepared MR angiography. Magn Reson Med 1996;36:384-392

61. Wilman AH, Riederer SJ, Breen JF, et al. Elliptical spiral phaseencoding order: an optimal, field-of-view-dependent ordering scheme for breath-hold contrast-enhanced 3D MR angioraphy. Radiology 1996;201(P):328-329

62. Maki JH, Prince MR, Chenevert TL. The effects of incomplete breath-holding on 3D MR image quality. JMRI 1997; 7:11321139

63. Snidow JJ, Aisen AM, Harris VJ, et al. Iliac artery MR angiography: comparison of three-dimensional gadolinium-enhanced and two-dimensional time-of-flight techniques. Radiology $1995 ; 196: 371-378$

64. Steiner P, Debatin JF, Romanowski B, et al. Optimization of breath-hold 3D MR pulmonary angiography. Radiology 1996;201(P):201

65. Hany TF, Debatin JF, Schmidt M, et al. Three-dimensional MR angiography of the renal arteries in a single breath-hold. Radiology 1996;201(P):218

66. Prince MR, Grist TM, Debatin JF. 3D contrast MR angiography. Berlin: Springer-Verlag, 1997:16-17

67. Strouse PJ, Prince MR, Chenevert TL. Effect of the rate of gadopentetate dimeglumine administration on abdominal vascular and soft-tissue MR imaging enhancement patterns. Radiology 1996;201:809-816

68. Prince MR, Chenevert TL, Foo TK, et al. Contrast-enhanced abdominal MR angiography: optimization of imaging delay time by automating the detection contrast material arrival in the aorta. Radiology 1997;203:109-114

69. Earls JP, Rofsky NM, DeCorato DR, et al. Breath-hold singledose gadolinium-enhanced three-dimensional MR aortography: usefulness of a timing examination and MR power injector. Radiology 1996;201:705-710

70. Krinsky G, Rofsky N, Flyer M, et al. Gadolinium-enhanced threedimensional MR angiography of acquired arch vessel disease. AJR 1996; 167:981-987

71. Foo TKF, Manojkumar S, Prince MR, et al. Automated detection of bolus arrival and initiation of data acquisition in fast, threedimensional, gadolinium-enhanced MR angiography. Radiology 1997; 203:275-280

72. Wilman AH, Debbins JP, Rossman PJ, et al. Flouroscopicallytriggered contrast-enhanced three dimensional MR angiography. Paper to be presented at the Fifth Scientific Meeting and Exhibition of the International Society for Magnetic Resonance in Medicine, Vancouver, Canada, 1997

73. Levy RA, Maki JH. Three-dimensional contrast-enhanced MR angiography of the extracranial carotid arteries: two techniques. AJNR 1998; 19:688-690 
74. Korosec FR, Grist TM, Frayne R, et al. Time-resolved contrastenhanced 3D MR angiography. Fourth Scientific Meeting and Exhibition. New York: International Society for Magnetic Resonance in Medicine, 1996:238

75. Korosec FR, Grist TM, Frayne R, et al. Time-resolved contrast-enhanced 3D MR angiography. Magn Reson Med 1996;36:345-351

76. Mistretta CA, Grist TM, Frayne R, et al. Contrast and motion artifacts in 4D MR angiography. Radiology 1996;201(P):238

77. Wood ML, Runge VM, Henkelman RM. Overcoming motion in abdominal MR imaging. AJR 1988; 150:513-522

78. Ehman RL, Felmlee JP. Adaptive technique for high-definition MR imaging of moving structure. Radiology 1989; 173:255-263

79. Du YP, Parker DL, Davis WL, et al. Reduction of partial-volume artifacts with zero-filled interpolation in three-dimensional MR angiography. JMRI 1994; 4:733-741

80. Ros PR, Gauger J, Stoupis C, et al. Diagnosis of renal artery stenosis: feasibility of combining MR angiography, MR renography, and gadopentetate-based measurements of glomerular filtration rate. AJR 1995; $165: 1447-1451$

81. Prince MR, Grist TM, Debatin JF. 3D contrast MR angiography. Berlin: Springer-Verlag, 1997:26-27

82. Laub G, Displays for MR angiography. Magn Reson Med $1990 ; 14: 222-229$
83. Rossnick S, Laub G, Braeckle R. Three dimensional display of blood vessels in MRI. Proceedings of the IEEE: computers in cardiology. New York: IEEE, 1986:193-195

84. Anderson C, Saloner D, Tsuruda J, et al. Artifacts in maximum intensity projection display of $\mathrm{MR}$ angiograms. AJR 1990; $154: 623-629$

85. Douek PC, Revel D, Chazel S, et al. Fast MR angiography of the aortoiliac arteries and arteries of the lower extremity: value of bolus-enhanced, whole-volume subtraction technique. AJR $1995 ; 165: 431-437$

86. Lee VS, Flyer MA, Weinreb JC, et al. Image subtraction in gadolinium-enhanced MR imaging. AJR 1996;167:14271432

87. Wang Y, Johnston DL, Breen JF, et al. Dynamic MR digital subtraction angiography using contrast enhancement, fast data acquisition, and complex subtraction. Magn Reson Med 1996; 36:551-556

88. Prince MR, Shoenberg SO, Ward JS, et al. Hemodynamically significant atherosclerotic renal artery stenosis: MR angiographic features. Radiology 1997; 205:128-136

89. Stafford-Johnson DB, Lerner CA, Prince MR, et al. Gadoliniumenhanced magnetic resonance angiography of renal transplants. Magn Reson Imaging, 1997; 15:13-20 